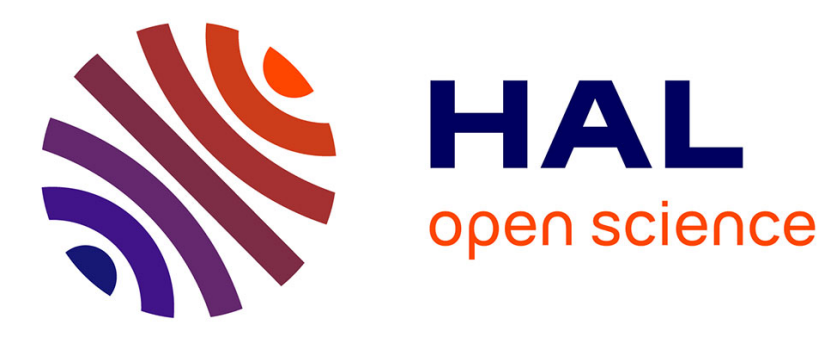

\title{
Competitive Adsorption between a Polymer and Solvents onto Silica
}

Julien Laurens, Julien Jolly, G. Ovarlez, Hélène Fay, Thomas Chaussée, Paul Sotta

\section{- To cite this version:}

Julien Laurens, Julien Jolly, G. Ovarlez, Hélène Fay, Thomas Chaussée, et al.. Competitive Adsorption between a Polymer and Solvents onto Silica. Langmuir, 2020, 36 (26), pp.7669-7680. 10.1021/acs.langmuir.0c01312 . hal-02995718

\section{HAL Id: hal-02995718 https://hal.science/hal-02995718}

Submitted on 10 Nov 2020

HAL is a multi-disciplinary open access archive for the deposit and dissemination of scientific research documents, whether they are published or not. The documents may come from teaching and research institutions in France or abroad, or from public or private research centers.
L'archive ouverte pluridisciplinaire HAL, est destinée au dépôt et à la diffusion de documents scientifiques de niveau recherche, publiés ou non, émanant des établissements d'enseignement et de recherche français ou étrangers, des laboratoires publics ou privés. 


\title{
Competitive Adsorption between a Polymer and Solvents onto silica
}

\author{
Julien Laurens, ${ }^{\dagger}$ Julien Jolly, $^{\dagger}$ Guillaume Ovarlez,${ }^{\dagger}$ Hélène Fay, ${ }^{\dagger}$ Thomas $^{-}$ \\ Chaussée, ${ }^{\ddagger}$ and Paul Sotta*, \\ $\dagger L O F$, University of Bordeaux, CNRS, Solvay, UMR 5258, 33608 Pessac, France \\ $\ddagger$ Solvay Silica, 15 rue Pierre Pays, 69660 Collonges au Mont dOr, France \\ ๆLaboratoire Polymères et Matériaux Avancés, CNRS, Solvay, UMR 5268, 87 avenue des \\ Frères Perret, 69192 Saint Fons Cedex, France \\ E-mail: paul.sotta-exterieur@solvay.com
}

\begin{abstract}
In polymer nanocomposites, particle-polymer interactions play a key role both in the processing and in the final properties of the obtained materials. Specifically, for silica, due to the surface polarity, surface modification is commonly used to improve the compatibility with apolar polymer matrices, in order to prevent agglomeration. In this work, a new way to investigating the polymer-silica affinity and determining dispersibility parameters (HDP) of silica particles in the 3D Hansen space using a solvent approach is proposed. These parameters are estimated from the assessment of the stability of suspensions in a set of organic solvents. Based on the respective locations of the solvent, polymer and silica representative points in the 3D Hansen space, the adsorption of a given polymer in solution in a given solvent can be predicted. This is shown with the industrial precipitated silica Zeosil ${ }^{\circledR} 1165 \mathrm{MP}$ in combination with polystyrene and polybutadiene. It is shown that silanization of the silica particles
\end{abstract}


decreases the adsorption of polystyrene, even though due to this surface treatment silica comes closer to polystyrene in the Hansen space. This counter-intuitive effect is rationalized based on the consideration of an adsorption parameter $\chi_{S}$ computed from the relative locations of the solvent, polymer and particles in the 3D Hansen space. Basically, this parameter is related to the respective distances of the solvent and polymer representative points to that of the particle in Hansen space.

\section{Introduction}

Silica nanoparticles are increasingly used as fillers in various types of polymer matrices in order to elaborate nanocomposite materials with enhanced properties for various applications. ${ }^{1}$ One prominent application is related to elastomer reinforcement ${ }^{2,3}$. It is nowadays well established that the performances of reinforced rubber materials used in tire applications can be enhanced by replacing carbon black by precipitated silica. ${ }^{4}$ Specifically, by tuning the morphologies of silica aggregates and the surface treatments, the silica-based reinforcing system can be tuned to optimize the compromise of key properties, rolling resistance vs wet grip. ${ }^{5,6}$ In terms of physical mechanisms, this is achieved by tuning the dissipation according to the range of frequency. ${ }^{7-9}$

The example of silica illustrates one of the key challenges that one has to face when dealing with polymer-silica nanocomposites. Polymers used in the tire industry are essentially nonpolar, i.e. hydrophobic, polymers, while silica nanoparticles, having hydroxyl groups at their surface, are hydrophilic entities. There is thus an issue to make silica compatible with the polymer, or at least dispersible within the polymer to a satisfactory degree. One advantage of silica is that it is suitable to surface treatment. Silica can be made hydrophobic to various degrees by grafting appropriate molecules ${ }^{10-13}$. In practical applications, reinforced elastomer materials for tires are most often processed by internal mixing. This requires, first, that silica nanoparticles themselves are elaborated in a form suitable to handle, on the

one hand, and to be dispersible in the polymer matrix down to the desired, nanometer or 
submicrometer scales, on the other hand. Then, the quality of the dispersion state and the performances, which are largely determined by it, are essentially driven by the process itself, namely by the complex, strongly non-linear flow applied in the internal mixer.

However, interactions at the polymer-silica interfaces also play a key role. Basically, efficient reinforcement of the final material requires good stress transmission through interfaces. Also, it has been shown that physical mechanisms which are essential to obtain strong reinforcement are related to the modification of the dynamical state of the polymer matrix in the vicinity of the filler particles, and this modification depends critically on the nature and strength of the interactions between the polymer and the filler particles ${ }^{14-18}$. During the process itself, even though it is driven by the shear rate and viscous stress applied in the mixer, it is of course crucial that filler particles or aggregates are compatible with the matrix, in the sense that they can be wetted by the polymer. Wettability is of course directly related to the nature of the interactions at interfaces, and polymer adsorption should be promoted.

Another class of materials and systems is rather processed by solvent casting. In this case, silica particles and the polymer matrix must first be dissolved or dispersed in a common solvent, which is then evaporated ${ }^{19-22}$. In this case, the dispersion state of the filler particles in the final material depend on the proper dispersion of the particles in the initial ternary mixture, as well as on the propensity of the particles to aggregate/agglomerate/segregate during the solvent casting step. For all these reasons, it is clear that it is crucial to get a comprehensive and quantitative understanding of the nature of the interactions between the polymer matrix of interest and silica nanoparticles or aggregates.

In order to study polymer/silica interactions, adsorption tests in organic media are often used $^{23,24}$. In this case the system is composed of three components: polymer, silica and solvent. While the polymer adsorption is considered to be a good indicator to understand the silica/polymer binary interaction, the nature of the solvent plays an important role in polymer adsorption as well ${ }^{25-32}$. It may affect the polymer conformation at interfaces and the dispersion state of the silica particles. There is quite little literature on the direct impact 
of the solvent on adsorption tests ${ }^{33-36}$. In general, the adsorbed polymer amount decreases when the solvent quality increases regarding the polymer-solvent pair interaction ${ }^{37-39}$. The substrate-solvent interaction is generally not explicitly considered.

From an alternative point of view, such ternary systems may be considered to generalize the concept of co-solvency, as the presence of a small amount of polymer in solution in a solvent may enhance the solubility of nanoparticles. However, specific aspects related to polymer adsorption are not simply described by the thermodynamics of solvents mixtures often considered to rationalize co-solvency effects. ${ }^{40}$

The work presented in this manuscript is focused on the impact of the polymer-solvent interaction on polymer adsorption on a given type of particle surface. The competition between solvent molecules and polymer chains onto the same surface is studied. It is shown that the preferential adsorption in the ternary system can be predicted using a generalized Hansen approach, based on quite basic considerations on pair interactions in molecular systems ${ }^{41}$. Some basic background on pair interactions and competitive adsorption in ternary systems is outlined in Section 2. Polystyrene and butadiene were selected because they are representative of polymer matrices used e.g. in the tyre industry to make reinforced elastomer materials. Indeed one often used polymer is SBR, a copolymer of styrene and butadiene.

There is little literature dealing with the determination of the Hansen parameters for insoluble solutes. In this case, it is more appropriate to call the parameters "Hansen Dispersibility Parameters" (HDP). Hansen dispersibility parameters have been used in the context of the compatibilization between carbon nanotubes and epoxy resin ${ }^{42,43}$. HDP have been determined for inorganic materials such as cerium oxides ${ }^{44}$ or zircon nanocrystals ${ }^{45}$ in order to optimize the dispersion state and assess the efficiency of the surface treatment. Hansen has proposed a method based on the preferential adsorption of various solvents on the same surface. ${ }^{46}$ Adsorption either leads to stabilization of the particles or favors aggregation. Based on these observations, some authors have proposed to discriminate the solvent 
quality by following the sedimentation ${ }^{47-49}$, based on the postulate that the sedimentation kinetics is directly affected by the attractive inter-particule interactions such as the Van der Waals forces or the hydrogen bonding capacity. ${ }^{50}$

\section{Background}

\subsection{Polymer/Solvent pair interactions}

The Flory interaction parameter $\chi_{F}$ is a dimensionless number which measures the enthalpy change per molecule in $k_{B} T$ units when a solvent (subscript $S$ ) and a monomer (subscript $P)$ molecules are exchanged from their respective pure phases. ${ }^{51}$ Based on the hypothesis that pair interaction forces are proportional to the product of quantities representative of each entity (namely the polarizability when e.g. van der Waals forces are considered) ${ }^{41}, \chi_{F}$ is proportional to the squared difference of the polarizabilities:

$$
\chi_{F} \approx \frac{z_{b}}{2 k_{B} T}\left(p_{S}-p_{P}\right)^{2}
$$

where $p_{i}$ is the polarizability of component $i, k_{B}$ the Boltzmann constant, $T$ the temperature and $z_{b}$ is some effective number of bonds through a small sphere which represents a monomer volume. Equivalently, the Flory interaction parameter can be expressed as:

$$
\chi_{F}=\frac{\sqrt{v_{m}^{(S)} v_{m}^{(P)}}}{k_{b} T}\left(\delta_{S}-\delta_{P}\right)^{2}
$$

where $v_{m}^{(i)}$ are the molecular volumes and $\delta_{i}$ the solubility parameters. The solubility parameter $\delta_{i}$ of a molecular substance $i^{52,53}$ is defined as the square root of the Cohesive Energy Density (CED), or enthalpy of vaporisation per unit volume $\Delta E_{i} / v_{m}^{(i)}$ where $\Delta E_{i}$ is the 
enthalpy of vaporization per molecule:

$$
\delta_{i}=\sqrt{\frac{\Delta E_{i}}{v_{m}^{(i)}}}
$$

The Flory theory describes incompressible solutions with no volume change upon mixing and with weak, non polar and non-directed (Van der Waals) interactions between species. In such conditions, according to Eq. $2, \chi_{F}$ is always positive. It corresponds in fact to the attractive contribution of the second virial coefficient in the corresponding polymer-solvent solution, which is expressed as $B_{2}=v_{m}(1-2 \chi) .{ }^{54}$ Within the framework of the Flory theory, the affinity in a polymer-solvent pair simply depends on the value of $\chi_{F}$. Good solvents correspond to $\chi_{F}<0.5$ (positive virial coefficient, monomer-monomer attractions are inhibited by the presence of solvent). The case $\chi_{F}=0$ is denoted athermal solvent. Conversely, poor solvents correspond to $\chi_{F}>0.5$ (negative virial coefficient, polymer-polymer attractions favored). The limit case $\chi_{F}=0.5$ corresponds to the so-called $\theta$ solvent case. For a given polymer-solvent pair, $\chi_{F}$ can be measured experimentally by various methods. $\chi_{F}$ is well defined for non-polar polymer/solvent systems and the predicted affinity is reasonably representative. Deviations are observed for solutes with high polarity or high hydrogen bonding capacity. ${ }^{55}$

In the Hansen Solubility Parameter (HSP) representation, the total solubility parameter is represented as a vector $\vec{\delta}$ with three components corresponding to the various contributions to intermolecular interactions, $\delta_{D}$ associated to dispersive contribution, $\delta_{P}$ to the polar contribution and $\delta_{H}$ to the hydrogen bonding capacity ${ }^{56-58}$. Each entity (solvent, subscript $S$, or monomer, subscript $P$ ) can then be represented as a point in the so-called 3D "Hansen space" where each axis represents a solubility contribution. The Flory interaction parameter is then directly related to the squared distance between the solvent and polymer 
representative points in the Hansen space:

$$
\chi_{F}=\frac{\sqrt{v_{m}^{(S)} v_{m}^{(P)}}}{k_{b} T}\left\|\overrightarrow{\delta_{S}}-\overrightarrow{\delta_{P}}\right\|^{2}
$$

It is very important to realize that Equation 4 and similar equations proposed below rely on the hypothesis that interaction energies associated to each type of intermolecular pair interactions (van der Waals and dipole-dipole interactions) contribute additively to the total interaction energy.

According to Hansen, the closer are the HSP values of the chosen solvent and the target polymer, the higher is the solubility. The notion of solubility of a polymer is thus extended to a sphere centered on the representative point of the polymer in the 3D Hansen space, with a radius corresponding to the $\theta$ solvent case $\chi_{F}=0.5$. All solvents located within this solubility sphere are good solvents, while points outside are bad solvents. A "Relative Energy Distance" (RED) parameter may be defined as the ratio of the polymer/solvent distance in the 3D Hansen space to the radius of the solubitility sphere. Solvents with $R E D<1$ shall then be good solvents of the considered polymer.

\subsection{Adsorption in a ternary system}

In this work, we only consider physisorption, in which no permanent covalent bond is established between the substrate surface and the polymer. When a polymer solution is put in contact with a surface, the polymer may adsorb on the surface depending on the balance of the polymer/solvent, polymer/surface and solvent/surface interactions ${ }^{59,60}$.

Let us consider a polymer $P$ in solution in a solvent $S$ in the presence of a surface $A .{ }^{41}$ Considering van der Waals forces, the opposite of the energy change when a molecule of solvent $S$ is replaced by a monomer $P$ on the surface $A$ can be expressed as follows ${ }^{41}$ :

$$
\chi_{S}=\frac{z}{2}\left(p_{P}-p_{S}\right)\left(p_{A}-p_{S}\right)
$$


where $p_{i}$ is the polarizabiliy of species $i$ and $z$ is some effective number of bonds through the occupied molecule area at the substrate surface.

One main hypothesis of this work is that the $\chi_{S}$ adsorption parameter can be used to predict the adsorption of a given polymer in solution in a given solvent.

Depending on the relative polarizability values of the various species, 3 cases may occur: 1) $p_{A}>p_{P}>p_{S}$. In this case $\chi_{S}$ is positive and the enthalpy change favors adsorption of polymer segments; 2) $p_{A}>p_{S}>p_{P} . \chi_{S}$ is negative and the adsorption of solvent molecule $S$ is favored, which leads to polymer depletion; 3) $p_{S}>p_{A}>p_{P}$. This case corresponds to the conditions of partial wetting with a contact angle of the polymer on the surface given by the Young's equation $\cos \theta=\left(p_{S}+p_{P}-2 p_{A}\right) /\left(p_{S}-p_{P}\right)^{41}$.

In the same way as for the Flory interaction parameter $\chi_{F}$ (Equation 4$), \chi_{S}$ may be expressed in terms of solubility parameters and its definition may be extended to the 3D Hansen space as:

$$
\chi_{S}=\frac{\sqrt{v_{m}^{(S)} v_{m}^{(P)}}}{k_{B} T}\left(\overrightarrow{\delta_{P}}-\overrightarrow{\delta_{S}}\right)\left(\overrightarrow{\delta_{A}}-\overrightarrow{\delta_{S}}\right)
$$

The factor $\left(\overrightarrow{\delta_{P}}-\overrightarrow{\delta_{S}}\right)$ describes the monomer/solvent affinity. Note that since $\chi_{S}$ is defined as a vector product, it may be negative if the vectors $\overrightarrow{\delta_{P}}-\overrightarrow{\delta_{S}}$ and $\overrightarrow{\delta_{A}}-\overrightarrow{\delta_{S}}$ go in opposite directions or make an angle larger than $90^{\circ}$. The second factor $\left(\overrightarrow{\delta_{A}}-\overrightarrow{\delta_{S}}\right)$ corresponds to the surface/solvent affinity. There is little literature on the binary interactions between solvents and a surface using a Hansen approach. In this paper we propose a new way to determine the Hansen contributions $\delta_{D}, \delta_{P}, \delta_{H}$ of a substrate based on a generalized Hansen approach (see section 3.3).

Note that in the above discussion we have implicitly assumed that the solid substrate has higher surface energy (proportional to $p_{A}{ }^{2}$ ) as compared to the polymer and solvent, which corresponds to the practical case considered here since silica has relatively large polar/hydrogen bond contributions, as will be assessed experimentally in what follows. 


\subsection{Adsorption of polymers}

Describing the adsorption of polymers on surfaces is a much more complex task than for molecular species, as adsorption involves a considerable change in the entropy of macromolecules and the associated contribution in the free energy may be largely dominant over enthalpic changes discussed above ${ }^{28,59,61}$. Nevertheless, comparing the polymer/solvent, polymer/surface and solvent/surface interaction energies remains crucial to predict the preferential adsorption ${ }^{61,62}$. A statistical theory taking into account in an explicit way the balance of interactions between polymer chains, solvent molecules and the substrate surface was proposed long ago ${ }^{25-27}$. Predictions were proposed for the evolution of the adsorbed polymer amount per unit surface $\Gamma$ as a function of the polymer concentration (i.e. for sorption isotherms) depending on the values of $\chi_{F}$ and $\chi_{S}$ (though only the cases of athermal $\left(\chi_{F}=0\right)$ and $\theta\left(\chi_{F}=0.5\right)$ solvents were considered $)$.

There are some importance differences with the present approach however. First and most important, the restrictive hypotheses that pair interactions are proportional to the product of polarisabilities of both entities, and that contributions of different types of interactions are additive, were not considered by Silberberg ${ }^{27}$. Instead, he assumes that the parameters $\chi_{F}$ and $\chi_{S}$ may vary independently, while in our case, from Eqs. 4 and 6, the condition $\chi_{F}=0$ (athermal solvent) implies $\chi_{S}=0$. Accordingly, a polymer in a good solvent should not adsorb in any case. Second, when translated in terms of polarizabilities using the above mentionned hypothesis, a slightly different definition of the adsorption parameter $\chi_{S}$ was used in $\operatorname{ref}^{27}$, namely $\chi_{S}=(z / 2)\left(p_{P}-p_{S}\right)\left(p_{A}-(1 / 2)\left(p_{P}+p_{S}\right)\right)$.

Figure 1 is reproduced from ref. ${ }^{27}$ and shows $\Gamma=f\left(\chi_{S}\right)$ curves for $\theta\left(\chi_{F}=0.5\right)$ and athermal $\left(\chi_{F}=0\right)$ solvents, for various polymer concentrations in the solution, for very long chains. It exhibits several qualitative features which are interesting to point out in the context of this work. Specifically it shows the strong effect of the solvent quality on polymer adsorption. The adsorbed amount is more than twice larger in $\theta$ solvent than in a good (athermal) solvent, that disfavors polymer adsorption. In all cases, at given $\chi_{F}$ 
and $\chi_{S}$ values, $\Gamma$ increases with the polymer concentration and reaches a plateau at large concentrations, in qualitative agreement with a Langmuir-type sorption isotherm shape. There is a minimum $\chi_{S}$ value below which no polymer adsorption occurs (dashed vertical lines in fig. 1). This value is larger in a good solvent, which disfavors polymer adsorption, than in $\theta$ solvent. More surprisingly, the adsorbed amount increases as $\chi_{S}$ decreases to values close to this minimum value. This is due to the presence of larger loops, as chains are very long and the number of segments directly adsorbed on the surface drops down in this regime of low $\chi_{S}$. At a given $\chi_{F}$, the adsorbed amount then tends to increase and reaches a plateau as $\chi_{S}$ increases.

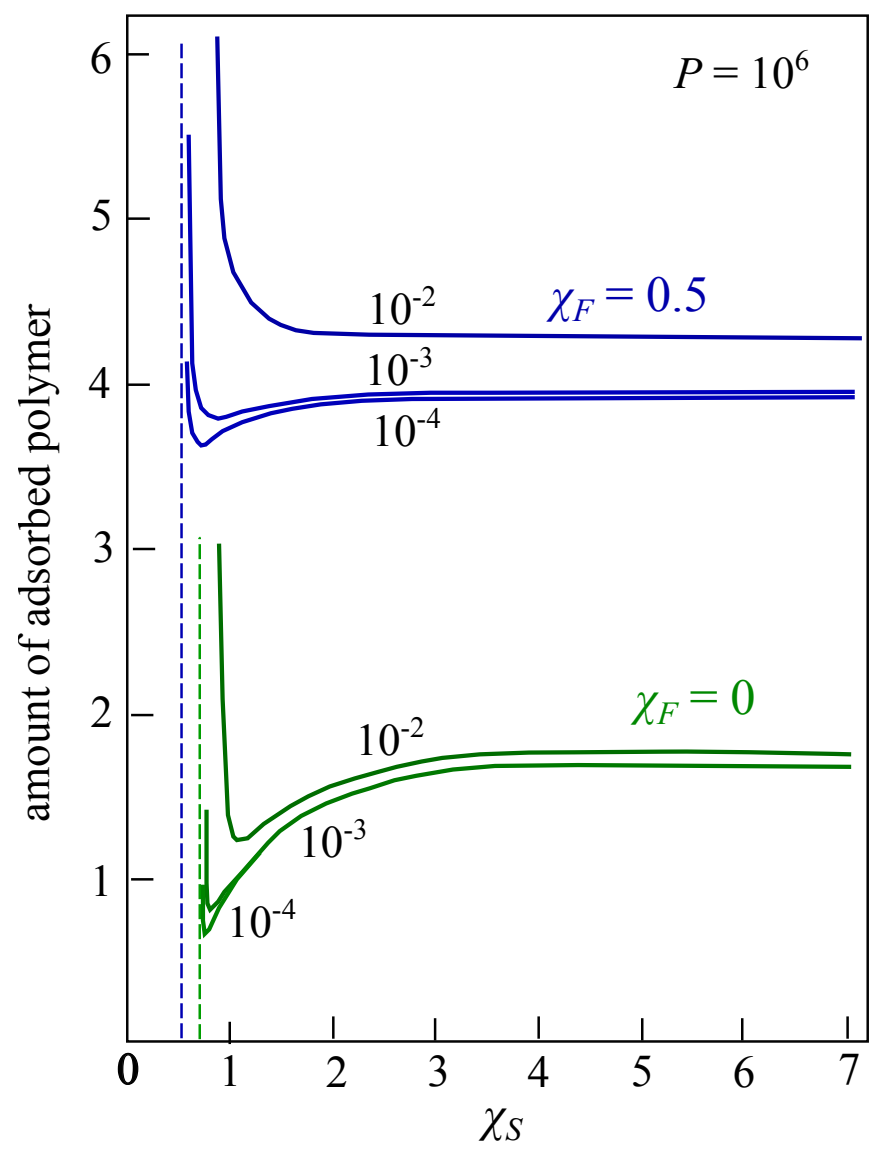

Figure 1: Amount of adsorbed polymer (in relative adimensional units) per unit surface as a function of the segmental adsorption parameter $\left(\chi_{S}\right)$ in a $\theta$ solvent $\left(\chi_{F}=0.5\right.$, blue curves) and in athermal solvent $\left(\chi_{F}=0\right.$, green curves) for different polymer volume fractions (as indicated on top of each curve), and for long chains (number of statistical segments $P=10^{6}$ as indicated). Adapted from Silberberg. ${ }^{27}$ 


\section{Experimental}

\subsection{Materials}

Industrial precipitated silica Zeosil ${ }^{\circledR}$ 1165MP (SOLVAY Silica, France) has been used in this work. The silica is processed in the form of a dispersible powder of grain size in the range of hundreds of $\mu \mathrm{m}$. In the fully dispersed state, Zeosil ${ }^{\circledR} 1165 \mathrm{MP}$ forms fractal aggregates of size around 50-60 $\mathrm{nm}$, themselves composed of elementary particles of $18 \mathrm{~nm}$. These aggregates are consolidated and have a rather compact structure with a mass fractal dimension of order 2.6 and a corresponding internal silica volume fraction of order 0.66 . The aggregates are agglomerated to form dispersible, so-called 'micropearl' structures of sizes in the range 100 $\mu \mathrm{m}$. This multiscale structure is schematized in figure 2 .

Silica was used as received and modified grades were obtained by silanisation. Triethoxy(octyl)silane (C8TEO) was used as covering agent to tune the silica surface properties. Anhydrous o-xylene (97\%) purchased from Sigma-Aldrich (Merk) was used for silanisation without further purification. The volume of o-xylene was adjusted to fix the silica concentration at $5 \mathrm{wt} \%$. The silane volume fraction, reaction time and temperature were controled in order to obtain different grafting ratios. The modified silica particles were separated by filtration of the suspension and washed by pure o-xylene to remove physisorbed C8TEO molecules. The separated solid phase was then dried at $60^{\circ} \mathrm{C}$ for $24 \mathrm{~h}$ and the grafted C8TEO amount was calculated from thermogravimetric analysis (TGA/DSC3+ STARe System - Mettler Toledo). The grafting ratio in C8TEO $X_{g r}$ is defined as the number of triethoxy units per square nanometer of bare silica surface. The $X_{g r}$ values for the four bare and modified silica grades are reported in Table 1. Specific surface areas (BET) were calculated for each obtained powder (Table 1) with a Gemini VII Micromeritics device, after dehydratation at $100^{\circ} \mathrm{C}$ during 3 hrs. Such a rather soft dehydratation process had to be used to prevent silane degradation in the case of grafted silica grades.

Monodisperse polystyrene PS170 (Alfa Aesar) Mw = $170000 \mathrm{~g} / \mathrm{mol}, \mathrm{Mw} / \mathrm{Mn}=1.06$ and 


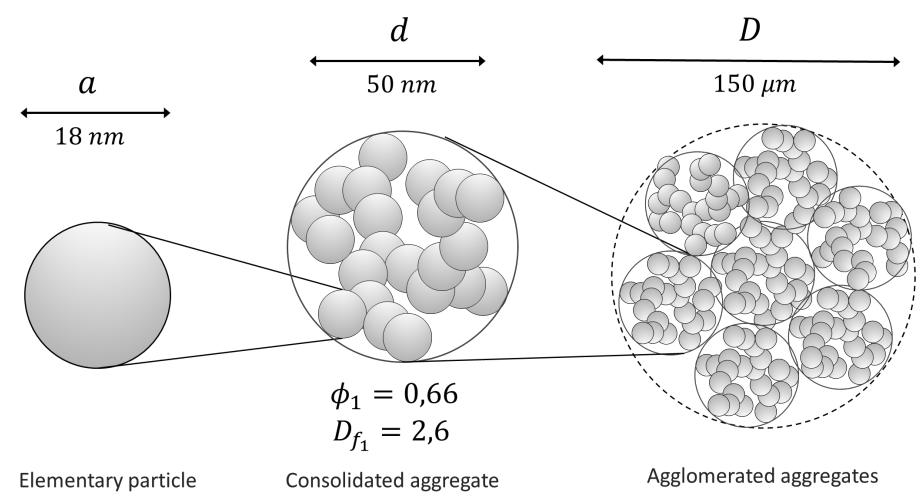

Figure 2: Multiscale structure of the precipitated silica Zeosil ${ }^{\circledR}$ 1165MP (not drawn to scale). Consolidated aggregates are the elementary structures to consider when dealing with silica dispersion in a solvent or polymer.

Polybutadiene BR200 (Sigma Aldrich - Merck) Mw $=200000 \mathrm{~g} / \mathrm{mol}, 36 \%$ cis-1,4, 55\% trans-1,4 and 9\% vinyl were used for adsorption isotherm measurements. All the solvents used in this study were analytical grades purchased from Sigma-Aldrich (Merck) and were used as received.

Table 1: Grafting ratios and BET specific areas for Zeosil ${ }^{\circledR} 1165 \mathrm{MP}$ silica samples neat and modified with triethoxy(octyl)silane. The denomination used for the samples are indicated in Column one.

\begin{tabular}{ccc}
\hline Sample & $X_{g r}\left(\right.$ silane $\left./ \mathrm{nm}^{2}\right)$ & Specific BET area $\left(\mathrm{m}^{2} / \mathrm{g}\right)$ \\
\hline S0 & 0 & 141 \\
S037 & 0.37 & 127 \\
S060 & 0.60 & 124 \\
S085 & 0.85 & 128 \\
\hline
\end{tabular}

\subsection{Hansen Solubility Parameters}

Hansen solubility parameters of polystyrene were estimated using solubility tests. The solubility of PS170 was analyzed at $20^{\circ} \mathrm{C}$ in about 60 solvents with various known HSPs (literature database $\left.{ }^{46}\right)$. The coordinates of the solvents are reported in the 3D Hansen space in Figure 3. The solvent volume was adjusted to maintain the polymer concentration at 5 wt\%. Visual inspection was performed after 24 hours of mixing at $20^{\circ} \mathrm{C}$. A good solvent is 
associated to a total solubility of the polymer (clear and transparent solution). Non-solvent are clearly identifiable for systems presenting no change in polymer aspect, i.e. corresponding to no dissolution at all. In some cases (intermediate solvents) the solubility is not total and the solvent-polymer mixture forms a turbid solution. The list of all solvents together with the corresponding HSP values and solubility scores is provided in Table S1.

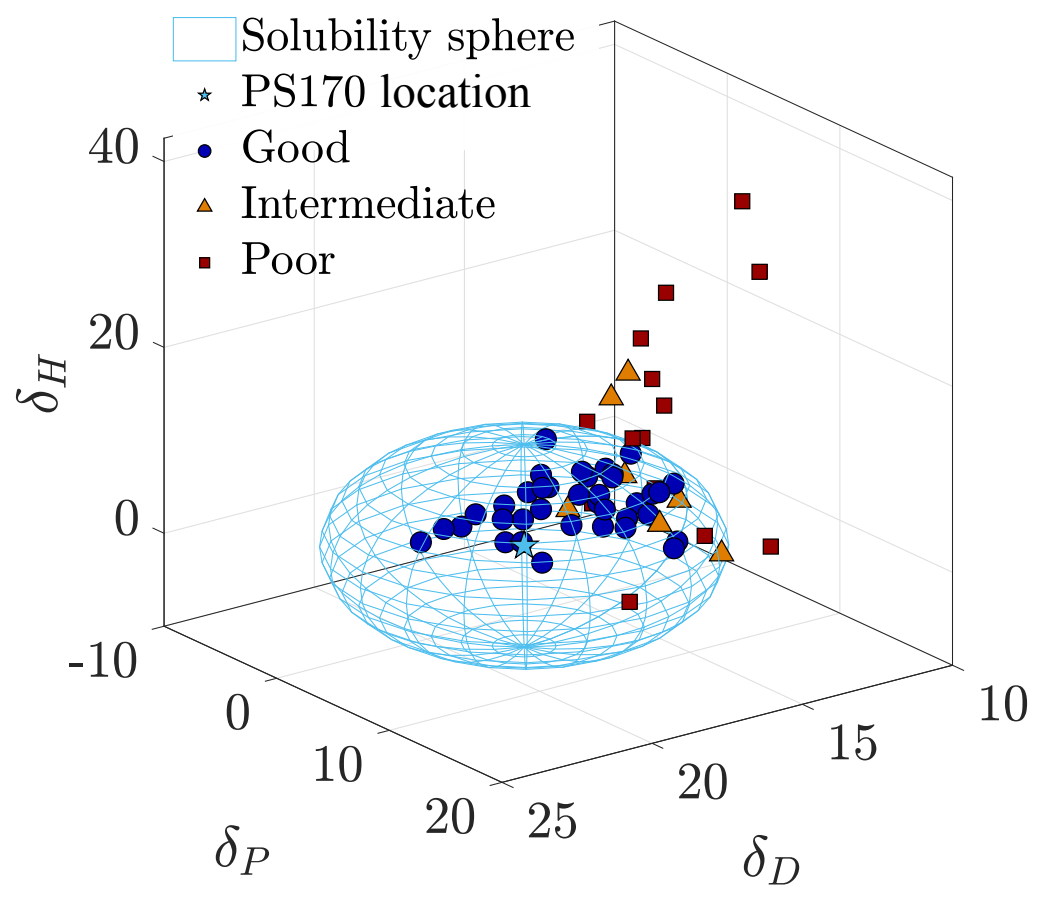

Figure 3: Determination of the solubility sphere of polystyrene in the 3D Hansen space. Different symbols indicate the HSP locations of the selected good $(\bullet)$, intermediate $(\Delta)$ and poor solvents $(\boldsymbol{\square})$. The PS170 location is identified as the center of the solubility sphere $(\star)$.

The solubility scores of the various PS/solvent solutions are indicated in figure 3 with different symbols/colors. Good solvents are represented with blue circles, intermediate solvents with orange triangles and non-solvents with red squares. A relatively clear frontier separates good and poor solvents. It can be noted that intermediate solvents are located in the close vicinity of this frontier. According to standard practice, the volume containing the locations of the good solvents is adjusted by a sphere (figure 3) and the the polymer HSP values are identified to the coordinates of the center of the sphere. The coordinates of PS170 
determined in this way are $\delta_{D}=19.42 \mathrm{MPa}^{1 / 2}, \delta_{P}=7.09 \mathrm{MPa}^{1 / 2}, \delta_{H}=3.54 \mathrm{MPa}^{1 / 2}$ and the radius $R_{\theta}$ of the sphere (corresponding to $\theta$ solvents) is equal to $10.9 \mathrm{MPa}^{1 / 2}$ in good agreement with the literature. ${ }^{46}$

\subsection{Hansen Dispersibility Parameters}

We propose to estimate the HDP values of our silica grades by evaluating the stability of silica suspensions in a set of various solvents, by measuring the sedimentation kinetics. In a good solvent, particles shall remain finely dispersed as they are of sub-micrometric size. Aggregation and sedimentation may occur on quite long time scales. Conversely, in a bad solvent, particles shall form larger aggregates, leading to faster sedimentation kinetics. A theoretical sedimentation rate can be related to the effective particle size using an extended Stokes equation:

$$
V_{\text {theo }}=\frac{2}{9} \frac{\Delta \rho g R_{0}^{2}}{\eta} f\left(\phi_{v}\right)
$$

where $\Delta \rho=\left(\rho_{p}-\rho_{s}\right)$ is the difference of the densities $\rho_{p}$ of the particles and $\rho_{s}$ of the solvent. $g=9.81 \mathrm{~ms}^{-2}$ is the gravitational acceleration, $R_{0}$ is the particle radius and $\eta$ the viscosity of the solvent. The function $f\left(\phi_{v}\right)=\left(1-\phi_{v}\right)^{5}$ takes into account the particle collision probability for spherical particles in non-diluted regime ${ }^{63,64}$.

First, particulate suspensions of concentration 2 wt\% were prepared in 22 different solvents. The list of solvents is provided in Table S2. The selected solvents present large variations in terms of $\delta_{D}, \delta_{P}$ and $\delta_{H}$. Glass beads were added in the suspensions to increase shear and the samples were placed in vortex agitation during two hours. This step of mechanical mixing is essential to break agglomerates and homogenize all systems. The typical size (diameter) of the obtained particles is $150 \mathrm{~nm} \pm 20 \mathrm{~nm}$ as measured by dynamic light scattering (Zetasizer Malvern). After this step the glass beads are removed and all 22 suspensions are placed in a dark chamber in front of a camera. A backlight is placed behind 
the samples to adjust the contrast.

Stability curves were measured at $20^{\circ} \mathrm{C}$. In order to measure the sedimentation kinetics, snapshots are taken every 5 to 10 seconds during 4 hours. Binarization and image analysis give space-time plots representing the relative sediment height $(R S H)$ as a function of time.

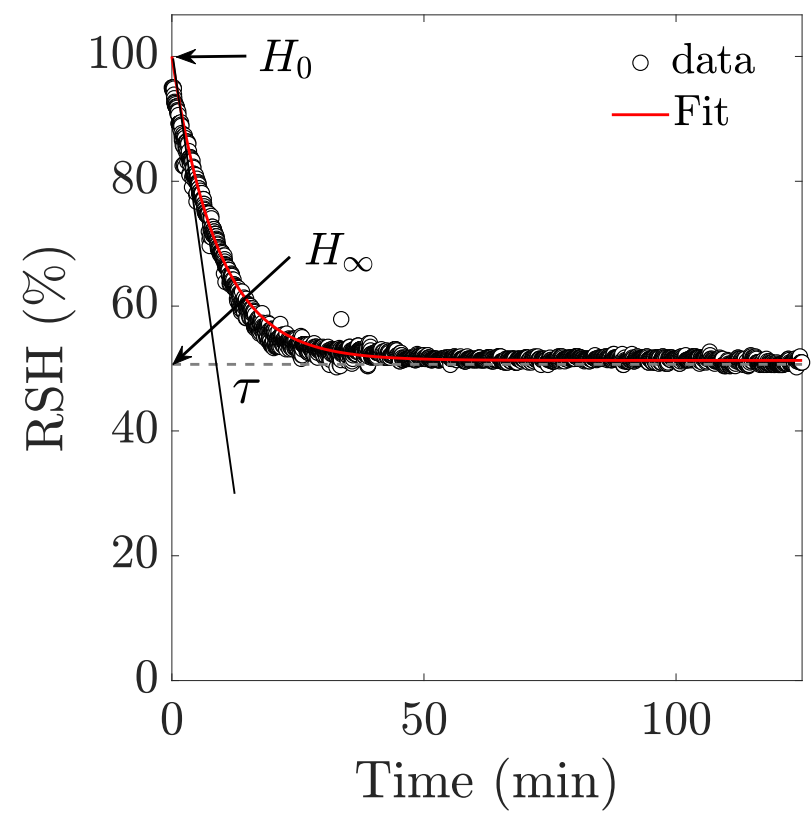

Figure 4: Determination of the stability curve.

Figure 4 shows a representative example of stability curve. A sharp decrease of $R S H$ is observed at short times followed by stabilization at a plateau value. Experimental data were fitted by a single exponential decay using equation 8 .

$$
R S H=H_{\infty}+\left(H_{0}-H_{\infty}\right) \exp (-t / \tau)
$$

$H_{0}$ corresponds to initial height of the suspension and $H_{\infty}$ to the final height of the sediment at equilibrium. The sedimentation time can be estimated with the $\tau$ parameter. The sedimentation rate $V_{\text {exp }}$ is computed from the sedimentation time $\tau$ as:

$$
V_{e x p}=\frac{\left(H_{0}-H_{\infty}\right)}{\tau}
$$


Relative suspension stabilities can be assessed by comparing the experimental and theoretical kinetics of each system. Based on these observations an indicator of stability $T_{c}$ may be proposed:

$$
T_{c}=\frac{V_{\text {theo }}}{V_{\text {exp }}}=\frac{\tau}{\left(H_{0}-H_{\infty}\right)} \frac{2}{9} \frac{\Delta \rho g R_{0}^{2}}{\eta}\left(1-\phi_{v}\right)^{5}
$$

For a good solvent, the experimental sedimentation kinetics $V_{e x p}$ is supposed to be very low and equal to the theoretical one $\left(V_{\text {theo }}\right)$, in such a way that $T_{c}$ should be equal to one. In suspensions in poor solvents, aggregation is clearly observed, particles form fractal agglomerates of radius $R_{e f f}$ eventually much larger than $R_{0}$ and the experimental sedimentation kinetics should be much faster than the theoretical one. In this case $V_{e x p}>>V_{\text {theo }}$ and $T_{c}$ tends to zero. In other words, $T_{c}$ is directly proportional to the squared ratio of the radius of the initial particles $\left(R_{0}\right)$ and the effective radius $\left(R_{e f f}\right)$ of the aggregated flocs:

$$
T_{c} \propto \frac{R_{p}^{2}}{R_{e f f}^{2}}
$$

$T_{c}$ is basically a dimensionless sedimentation timescale normalized between 0 (poorest solvent) and 1 (best solvent). For example, in linear alkanes such as heptane, the destabilization kinetics of is very fast, resulting in a very low $T_{c}=6.7 \times 10^{-5}$. Using this value in equation 11 the effective agglomerate radius is found to be around $9 \mu \mathrm{m}$, while it is about $150 \mathrm{~nm}$ in good solvent. Note also that the radius $R_{\text {eff }}$ should not be strictly identified with the overall size of the fractal aggregates, as the density of the solid phase (aggregates) changes during aggregation. In order to better distribute $T_{c}$ values, a stability coefficient $S_{\text {coef }}$ based on a logarithmic scale was introduced:

$$
S_{\text {coef }}=-\frac{\log \left(T_{c}\right)}{\min \left(\log \left(T_{c}\right)\right)}+1
$$

where $\min \left(\log \left(T_{c}\right)\right)$ is the minimum value observed in the whole set of solvents. In this new scale, the best solvent corresponds to $S_{\text {coef }}=1$ and the poorest solvent to 0 . All stability 
curves are shown in Figure S1. The stability coefficients $S_{\text {coef }}$ calculated from these curves for all silica/solvent systems are reported in Table S2.

It can be noted that the equilibrium final heights $\left(H_{\infty}\right)$ depend on the solvent quality. The final height is between $91.8 \%$ and $56.6 \%$. This behavior could be due to differences in agglomerate conformations. Indeed in organic media, silica aggregation is strongly affected by the solvation forces and drive the sedimentation phenomenon. We suppose that for long times the packing is more or less dense due to the difference on elementary fractal conformations.

\subsection{Adsorption isotherms}

Adsorption isotherms were measured in dilute organic solution at $35^{\circ} \mathrm{C}$. After polymer solubilization, silica is added at $5 \mathrm{wt} \%$. The samples were magnetically stirred during 24 hours. After this period, the mixture was centrifuged and the supernatant was removed. The obtained silica was then washed with pure solvent and centrifuged again 4 times in order to remove the non-adsorbed polymer. The silica was then dried at $40^{\circ} \mathrm{C}$ under vacuum for 48 hrs. The obtained powder was then analysed by ThermoGravimetric Analysis (TGA). The amount $\Gamma$ of adsorbed polymer (in $\mathrm{mg} . \mathrm{m}^{-2}$ ) was calculated from the weight loss measured in TGA between 300 and $600^{\circ} \mathrm{C}$ using equation 13 :

$$
\Gamma=\frac{\Delta m_{p}}{\left(m_{s}-m_{w}\right) S_{B E T}}
$$

Note that in all cases the weight loss is centered around $360^{\circ} \mathrm{C}$, the same temperature as measured for pure polystyrene. Polystyrene solutions were prepared with concentrations $2,4,6,10,20$ and $40 \mathrm{mg} / \mathrm{ml}$ in order to obtain the adsorption isotherms. The obtained adsorbed amounts were fitted as a function of the polymer concentration using a Langmuir sorption isotherm model:

$$
\Gamma=\frac{\Gamma_{\max }^{l} K_{c} C_{\text {polym }}}{1+K_{c} C_{\text {polym }}}
$$


The two fitting parameters extracted from eq.14 are the maximum adsorbed amount $\Gamma_{\max }^{l}$, which corresponds to the plateau value at high polystyrene concentrations, and the adsorption constant $K_{c}$.

\section{Results and discussion}

We first present experiments which were performed to locate the bare and modified silica in the 3D Hansen space. We then discuss polymer adsorption on bare silica in terms of the polymer-solvent affinity. We finally show that, for discussing the effect of the silica surface treatment (silanization), the adsorption parameter $\chi_{S}$ as defined in Section 2.2 must be considered.

\subsection{Hansen Dispersibility Parameters of silica, impact of surface treatment}

Figure 5 shows 3D representations of the solvent dataset used for the stability tests of all silica samples. For each solvent, the stability coefficient (see Table S2) is indicated by a color code varying between red (poor solvents) and blue (good solvents) according to the indicated color bar. This new approach allows introducing a non-binary criterium deduced from the stability curves.

Several methods may be used to compute Hansen dispersibility parameters (HDPs) of the substrates from the sets of data shown in Figure 5. Howell et al. have proposed to adjust the volume defined by the good solvents (in our case where $S_{\text {coef }}=1$ ) using a convex hull. ${ }^{65}$ Such convex hulls are represented in figure 5 for our systems. A clear frontier appears between the good solvents $\left(S_{\text {coef }}=1\right)$ and the other ones. The good solvent region seems to be convex, as no poor solvent is located in the stability region.

Several different ways of determining the centers of these volumes may then be used in order to estimate the HDPs of the various silica grades. ${ }^{65} \mathrm{~A}$ first way is to compute the 
centroid of each convex volume, treating it as a solid with uniform density. ${ }^{65}$ The representative points of the silica samples using the centroid calculation method are plotted in figure 5 with open symbols. Another way is to use a barycentric method, computing the location of a silica sample as the mass center of the whole dataset (including bad solvents) weighted by the stability coefficients:

$$
\delta_{i}=\frac{\sum_{j} S_{c o e f}^{(j)} \delta_{i}^{(j)}}{\sum_{j} S_{c o e f}^{(j)}}
$$

The representative points of the silica samples obtained with the barycentric method are indicated in Figure 5 with black symbols. At the end, we propose to adopt as final Hansen dispersibility parameters the mean of the points obtained by the barycenter and centroid methods. The results are summarized in Table 2 and in Figure 5. The 2D projections in the $\left(\delta_{P}, \delta_{H}\right)$ plane of the convex hulls obtained for the various silica grades are shown in Figure $\mathrm{S} 2$.

Table 2: HDP values of the neat (S0) and triethoxy(octyl)silane-modified Zeosil ${ }^{\circledR} 1165 \mathrm{MP}$ silica samples.

\begin{tabular}{cccc}
\hline Sample & $\delta_{D}\left(\mathrm{MPa}^{1 / 2}\right)$ & $\delta_{P}\left(\mathrm{MPa}^{1 / 2}\right)$ & $\delta_{H}\left(\mathrm{MPa}^{1 / 2}\right)$ \\
\hline S0 & $16.34 \pm 0.17$ & $12.09 \pm 1.63$ & $13.84 \pm 1.57$ \\
S037 & $16.33 \pm 0.19$ & $11.80 \pm 1.89$ & $13.15 \pm 1.57$ \\
S060 & $16.44 \pm 0.19$ & $10.50 \pm 1.30$ & $9.41 \pm 1.57$ \\
S085 & $16.46 \pm 0.18$ & $10.21 \pm 1.70$ & $9.13 \pm 1.57$ \\
\hline
\end{tabular}

In view of experimental data reported in Table 2, the evolution of HDPs as a function of the silane grafting ratio can be discussed. The $\delta_{D}$ contribution is not significantly affected by the presence of the covering agent. It is independent of the grafting ratio. The $\delta_{P}$ and $\delta_{H}$ contributions are affected when the surface is modified. Indeed, when the grafting ratio increases the polar and hydrogen bonding contributions decrease. For the unmodified silica $\delta_{P}$ is equal to $12.09 \mathrm{MPa}^{1 / 2}$ and decreases down to $10.21 \mathrm{MPa}^{1 / 2}$ for the highest modified silica sample. For the $\delta_{H}$ contribution the decrease is more than $4.7 \mathrm{MPa}^{1 / 2}$ between bare silica and the highest modified sample. This result reflects the general loss of polarity and 

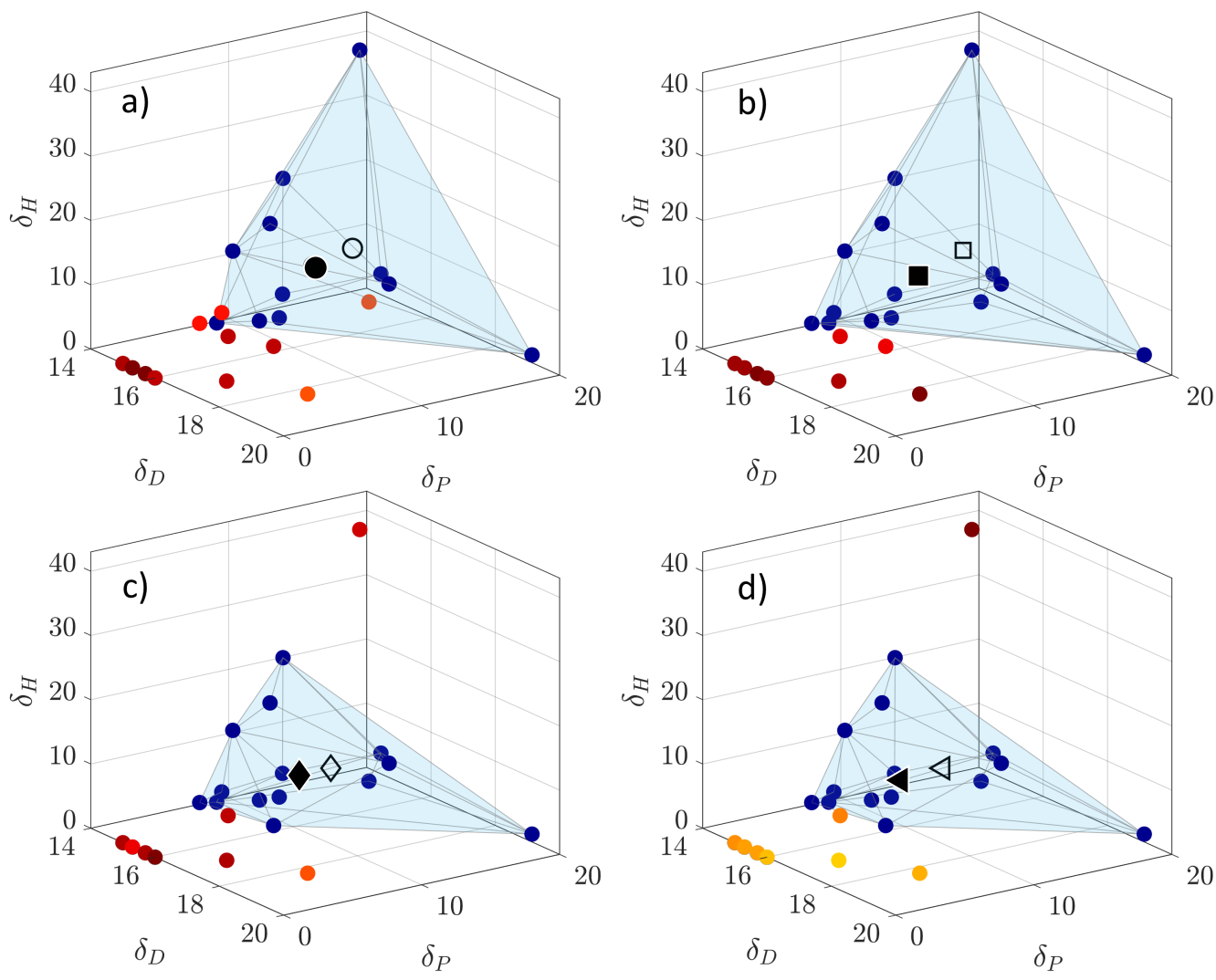

Poor dispersibility

(Gel)

$S_{\text {coef }}$

Good dispersibility

(Suspension)

Figure 5: Representation of the silica-solvent systems in the Hansen 3D space. Solvent representative points are colored according to the stability coefficient value. Calculated silica locations are represented: a) Bare Z1165MP silica (S0) (circles); b) S037 (squares); c) S060 (diamonds); d) S085 (left triangles); Hollow symbols: centroid calculation method; Black symbols: barycentric method.

hydrogen bonding capacity of the silica surface. The unmodified silica has the maximum density of free silanols, which explains the highest $\delta_{P}$ and $\delta_{H}$ values. The silanisation reaction takes place on free silanols and forms siloxane bonds with the covering agent, i.e. it induces a decrease of the free silanol density. The measured evolution of the HDPs thus demonstrates the robustness of the HDP calculation using the proposed method.

The HSPs of polystyrene and the HDPs of the bare and C8TEO-modified Z1165MP silica grades are shown in Figure 6 in the 3D Hansen representation. Errors bars indicated for the 
silica points correspond to the differences between the centroid and barycentric estimates of the locations of silica representative points. The representative points of the modified silicas moves towards the region in the Hansen space corresponding to less polar and less hydrogen-bonded entities as the grafting ratio increases. As a results, it comes closer to the polystyrene HSP values, as illustrated in fig.6 by the dashed arrow. This shift should indicate an increase of the polymer/silica affinity.

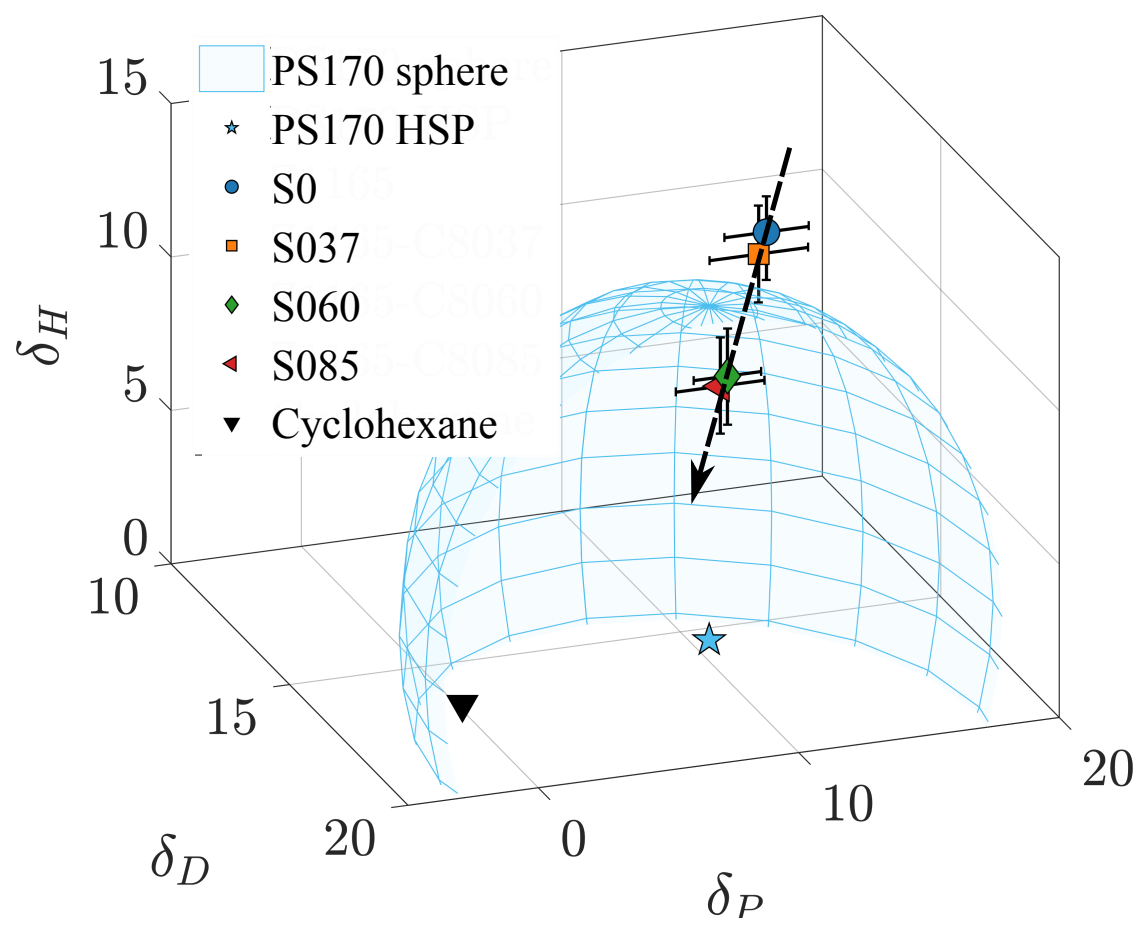

Figure 6: Representation of the polystyrene HSPs $(\star)$ and of the HDPs of modified silica

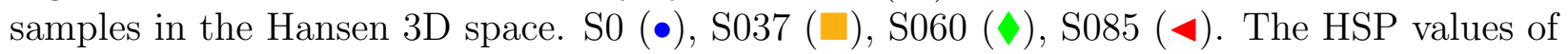
cyclohexane are also represented $(\boldsymbol{\nabla})$. The dashed arrow indicates the trend as the silica is increasingly treated.

\subsection{Adsorption isotherms, solvent effect}

In order to investigate the effect of the solvent on polymer adsorption on silica, polystyrene adsorption isotherms were measured in various solvents with different HSPs (Table 3). The selected solvents were (in order of decreasing RED with PS) cyclohexane, diethyl-malonate, 
1-chlorobutane, anisole and 1,4-dichlorobutane. The polar and hydrogen bonding Hansen components of cyclohexane are close to zero as compared to 1-chlorobutane or anisole, which have $\delta_{P}$ and $\delta_{H}$ values larger than $5 \mathrm{MPa}^{1 / 2}$. This large range of $\mathrm{HSP}$ values shall change the $\chi_{S}$ value and thus affect polymer adsorption. The polymer/solvent $R E D$ values (see Section 2.1) are reported in Table 3. While all solvents are located inside the solubility sphere of PS $(R E D<1)$ in order to solubilize the polymer, the Flory parameter $\chi_{F}$ changes for the various solvents. The best solvent is anisole with the lowest $R E D$ equal to 0.49 while cyclohexane is close to $\theta$ solvent with the highest $R E D=0.87$. The positions of solvents and the PS sphere are represented in Figure 7, together with the silica HDP values.

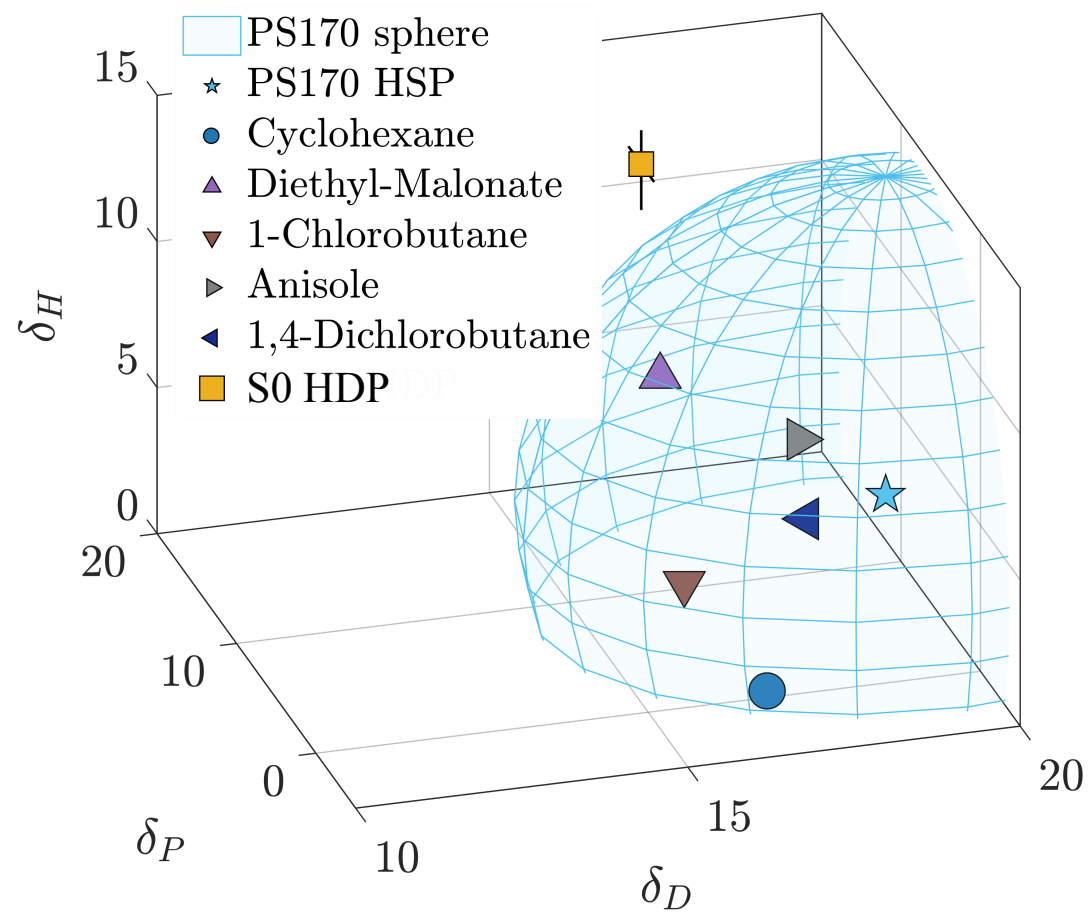

Figure 7: Representation of the HSP values of polystyrene $(\star)$ and of the studied solvents and of the HDP values of the bare Z1165MP silica (S0) ( $\square$ ) in the Hansen 3D space: Cyclohexane $(\bullet)$, Diethyl-Malonate $(\boldsymbol{\Delta})$, 1-Chlorobutane $(\boldsymbol{\nabla})$, Anisole $(\boldsymbol{\bullet})$ and 1,4-dichlorobutane $(<)$.

In this set of solvents, polystyrene adsorption was measured on the bare silica S0. The adsorption isotherms are shown in Figure 8 a). It is clear that the polymer adsorption 
Table 3: HSP values of the selected solvents and RED distance from polystyrene.

\begin{tabular}{lcccc}
\hline Sample & $\delta_{D}\left(\mathrm{MPa}^{1 / 2}\right)$ & $\delta_{P}\left(\mathrm{MPa}^{1 / 2}\right)$ & $\delta_{H}\left(\mathrm{MPa}^{1 / 2}\right)$ & $R E D(P S 170)$ \\
\hline Cyclohexane & 16.8 & 0 & 0.2 & 0.87 \\
Diethyl-Malonate & 16.1 & 7.7 & 8.3 & 0.75 \\
1-Chlorobutane & 16.2 & 5.5 & 2 & 0.62 \\
Anisole & 17.8 & 4.4 & 6.9 & 0.49 \\
1,4-dichlorobutane & 18.3 & 7.7 & 2.8 & 0.22 \\
\hline
\end{tabular}

strongly depends on the solvent quality. In all cases the curves are qualitatively similar to Langmuir adsorption isotherms, with a relatively sharp increase of $\Gamma$ at low polymer concentrations and then a stabilisation at a plateau value. Adsorption parameters obtained by fitting sorption isotherms with the Langmuir model are reported in Table 4 . To illustrate this dependence, the maximum adsorption of PS170 is plotted in Figure 8 b) as a function of the polymer/solvent $R E D$ value. By adjusting the quality of the solvent, the adsorbed amount can be tuned from a high value (a few $\mathrm{mg} / \mathrm{m}^{2}$ ) down to nearly zero.

This observation is in good qualitative agreement with Figure $1 .{ }^{27}$ The adsorption is higher in theta solvent $\left(\chi_{F}=0.5, R E D\right.$ values close to 1$)$ than in athermal solvent $\left(\chi_{F}=0\right.$, small $R E D)$.

Note that some residual adsorption is still measured in the case of good solvents (RED $\leq$ 0.5 ), with perhaps a tendency to increasing adsorption at very low $R E D$ (or equivalently $\chi_{F}$ ) values (case of 1,4-dichlorobutane). At low $\chi_{F}$ values, the fraction of monomers in contact with the surface should be very small, which, for long chains, may induce the presence of very long loops and tails. This effect may lead to an increase of the thickness of the adsorbed polymer layer and may possibly compensate the decrease of the number of adsorbed monomers. This unexpected behavior at small $\chi_{F}$ values may also be due to a change in the solvent affinity with the silica surface. Indeed, the solvent/surface interaction was not considered in this representation and it may be easily assumed that the solvent/silica affinity may be different for different solvents. The importance of taking the solvent effect into account will be discussed in more details in Sections 4.3 and 4.4. 
a)

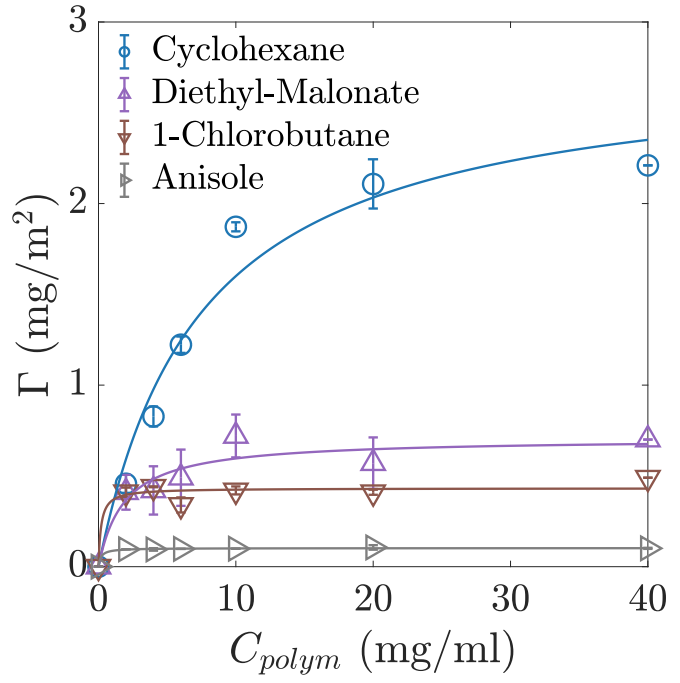

b)

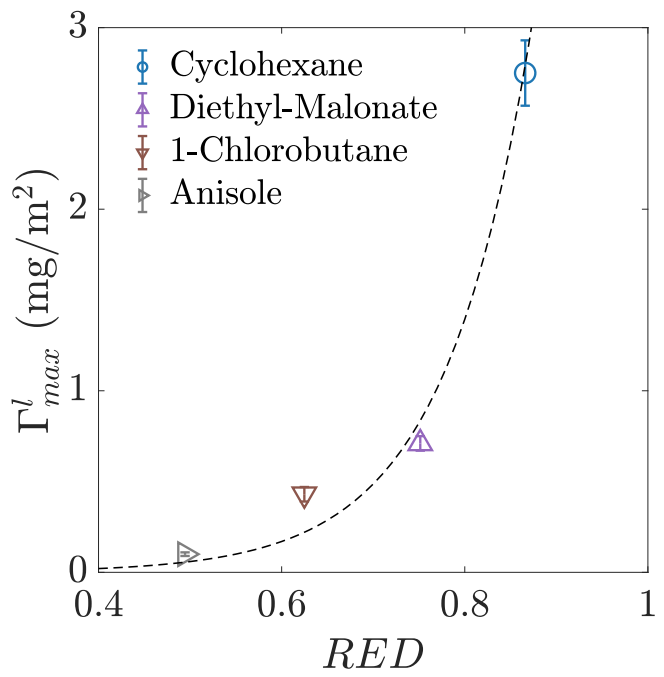

Figure 8: a) Adsorption isotherms of PS170 on the untreated S0 silica in cyclohexane (०), diethyl-malonate $(\triangle)$, 1-chlorobutane $(\nabla)$ and anisole $(\triangleright)$. b) The maximum adsorbed amount as a function of the solvent/polymer $R E D$ distance. The dashed curve is a guide for the eye.

Table 4: Langmuir fit parameters $\Gamma_{\max }^{l}$ and $K_{c}$ for adsorption isotherms of PS170 on S0.

\begin{tabular}{lcc}
\hline Sample & $\Gamma_{\max }^{l}\left(\mathrm{mg} / \mathrm{m}^{2}\right)$ & $K_{c}$ \\
\hline Cyclohexane & $2.75 \pm 0.18$ & $0.14 \pm 0.09$ \\
Diethyl-Malonate & $0.71 \pm 0.04$ & $0.54 \pm 0.10$ \\
1-Chlorobutane & $0.43 \pm 0.04$ & $5.43 \pm 0.07$ \\
Anisole & $0.10 \pm 0.01$ & $5.72 \pm 0.05$ \\
1,4-dichlorobutane & $0.29 \pm 0.01$ & $1.47 \pm 0.05$ \\
\hline
\end{tabular}

\subsection{Competitive adsorption}

A further experiment, in which the adsorbed amounts of two different polymers in two different solvents are compared, may be performed in order to assess the primary role of polymer-solvent affinity. Two polymers, the PS170 polystyrene and the BR200 polybutadiene, were used for this test. The solubility sphere of BR200 was experimentally determined using the method described in section 3.2. The HSP values of BR200 are $\delta_{D}=18.66 \mathrm{MPa}^{1 / 2}$, $\delta_{P}=0.16 \mathrm{MPa}^{1 / 2}$ and $\delta_{H}=3.86 \mathrm{MPa}^{1 / 2}$ and the radius of the sphere is equal to $9.40 \mathrm{MPa}^{1 / 2}$. Being non-polar, Polybutadiene has low polar and hydrogen bonding contributions. The sol- 
ubility spheres are shown in Figure 9 for both polymers. The representative point of the bare silica $\mathrm{S} 0$ is also reported in Figure 9.

The adsorption isotherms of these two polymers were measured in cyclohexane and 1,4dichlorobutane, which were selected due to their specific locations in Hansen space. The HSPs of the solvents are reported in figure 9. Both solvents are located inside the overlap of the two solubility spheres and they solubilize both polymers. Cyclohexane is close to BR200, while 1,4-dichlorobutane is close to PS170. All polymer/solvent $R_{a}$ distances are summarized in Table 5. Thus, these two solvents were chosen to reverse the ratio of the polymer/solvent distances, with $R_{a}(\mathrm{BR} 200)<R_{a}(\mathrm{PS} 170)$ in cyclohexane and $R_{a}(\mathrm{PS} 170)$ $<R_{a}(\mathrm{BR} 200)$ in 1,4-dichlorobutane.

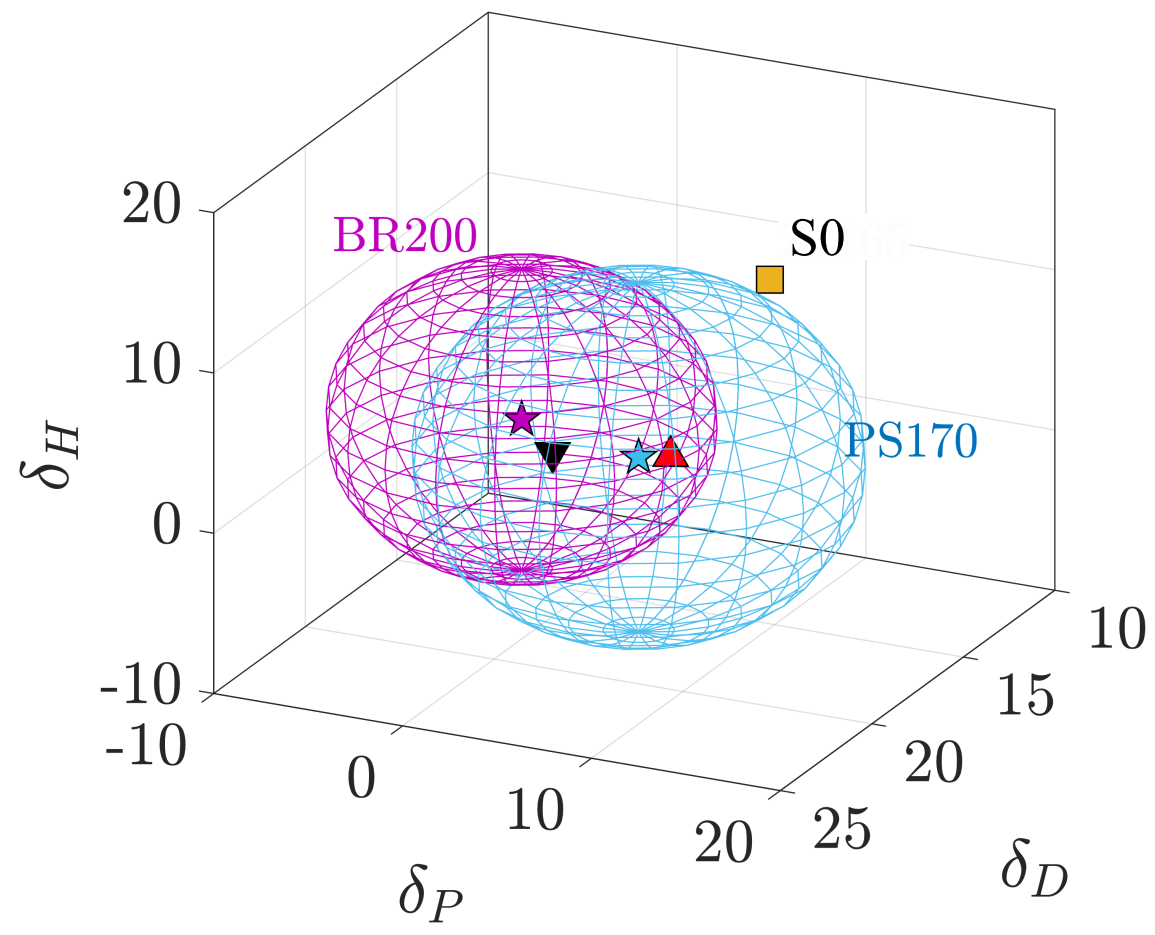

Figure 9: Representation of the HSP values of polystyrene $(\star)$, polybutadiene $(\star)$, cyclohexane $(\boldsymbol{\nabla})$ and 1,4-dichlorobutane $(\boldsymbol{\Delta})$ and of the HDP values of the S0 silica $(\square)$ in the Hansen 3D space.

Adsorption isotherms of PS170 and BR200 in these two solvents are shown in figure 
10. In cyclohexane (full curves in figure 10) the adsorption of PS170 is more than twice larger than that of BR200 (ratio of 0.36). $\Gamma_{\max }^{l}$ is equal to $1.00 \pm 0.01 \mathrm{mg} / \mathrm{m}^{2}$ for BR200 and $2.75 \mathrm{mg} / \mathrm{m}^{2}$ for PS170. In the proposed approach, this difference originates in the short BR200/cyclohexane distance, i.e. the strong polymer/solvent affinity for this pair, as described in section 4.2. In 1,4-dichlorobutane (dashed curves in figure 10) the relative positions of adsorption isotherms are reversed. In this solvent, polybutadiene adsorption is now larger than that of polystyrene. The adsorption plateau values (Table 5) are $0.29 \pm$ $0.01 \mathrm{mg} / \mathrm{m}^{2}$ for PS170 and $0.95 \pm 0.13 \mathrm{mg} / \mathrm{m}^{2}$ for BR200.

Therefore this result confirms that the polymer/solvent affinity has a major impact on the adsorption and that the inversion of preferential adsorption of two different polymers on the same surface can be predicted on the basis of the relative positions of the solvents. When changing the solvent, the ratios between the $\Gamma_{\max }^{l}$ of the most and less adsorbing polymers remain similar. However, absolute adsorption values are lower. This decrease may be explained by the shorter polymer/solvent distances in 1,4-dichlorobutane than in cyclohexane, as shown in Table 5.

Table 5: HSP distances between PS170, BR200 and cyclohexane and 1,4-dichlorobutane and $\Gamma_{\max }^{l}$ values determined from the data in Figure 10 for the different systems.

\begin{tabular}{lccc}
\hline & Solvents & PS170 & BR200 \\
\hline$R_{a}$ distance $\left(\mathrm{MPa}^{1 / 2}\right)$ & Cyclohexane & 9.50 & 5.36 \\
& 1,4-Dichlorobutane & 2.54 & 7.65 \\
\hline$\Gamma_{\max }^{l}\left(\mathrm{mg} / \mathrm{m}^{2}\right)$ & Cyclohexane & $2.75 \pm 0.18$ & $1.00 \pm 0.01$ \\
& 1,4-Dichlorobutane & $0.29 \pm 0.01$ & $0.95 \pm 0.13$ \\
\hline
\end{tabular}

\subsection{Adsorption isotherms, impact of the silica surface treatment}

Adsorption isotherms of polystyrene on bare and silanized silica samples were measured in cyclohexane, which is a $\theta$ solvent $\left(\chi_{F}=0.5\right)$ for polystyrene at $35^{\circ} \mathrm{C}$. The representative point of cyclohexane is also reported in the 3D Hansen space in fig.6. 


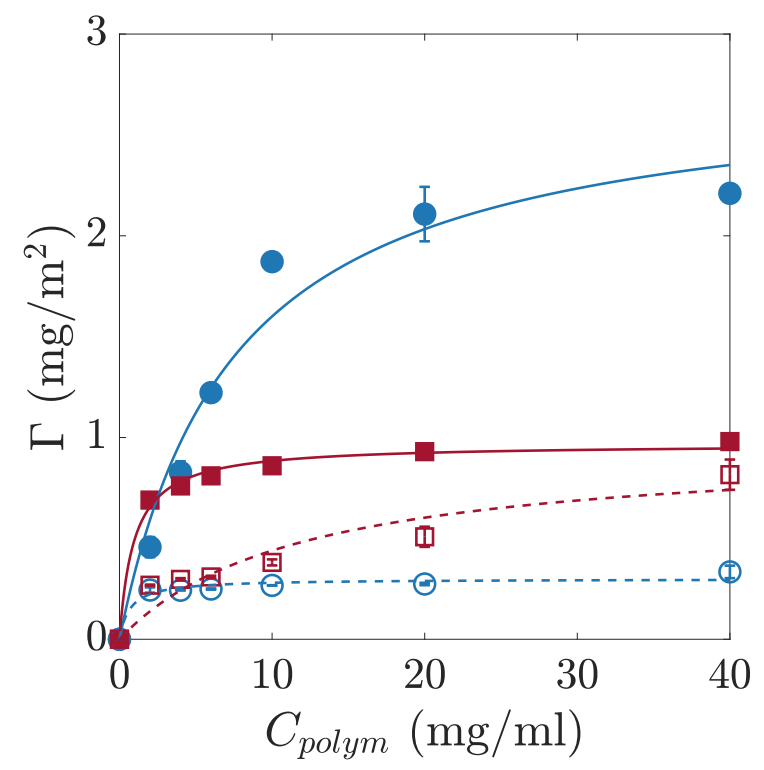

Figure 10: Adsorption isotherms of PS170 (•) and BR200 (ם) on the bare Zeosyl ${ }^{\circledR} 1165 \mathrm{MP}$ silica in cyclohexane (full curves). Adsorption isotherms of PS170 (o) and BR200 ( $\square$ ) on S0 silica in 1,4-dichlorobutane (dashed curves).

The adsorption isotherms of polystyrene on the various silica samples in cyclohexane are shown in Figure 11 a). Fitted parameters $\Gamma_{\max }^{l}$ and $K_{c}$ are reported in Table 6.

a)

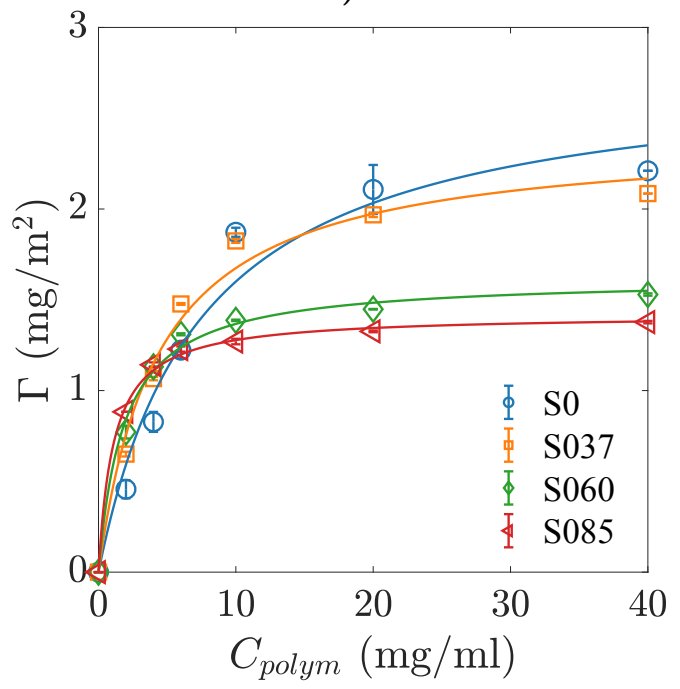

b)

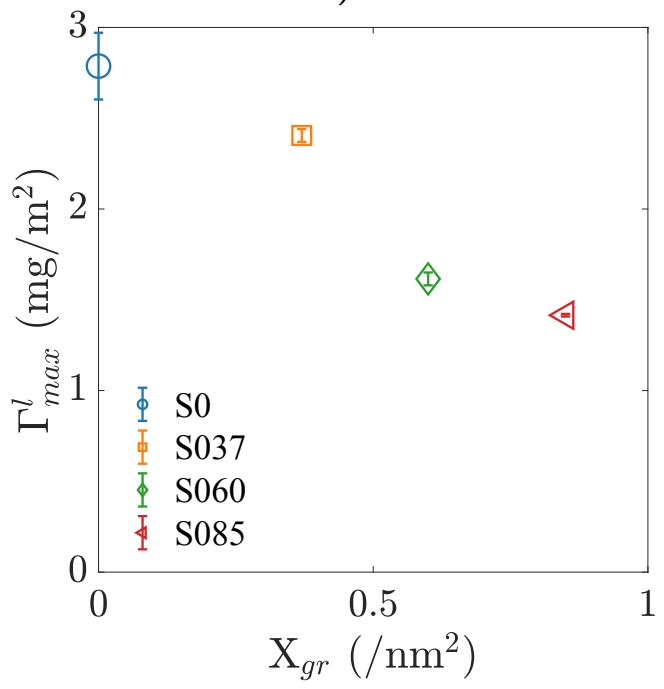

Figure 11: a) Adsorption isotherms of PS170 on the S0 silica (०), on S037 ( $\square)$, on S060 $(\triangleright)$ and on S085 $(\triangleleft)$. b) The maximum adsorbed amount as a function of the grafting ratio. 
Table 6: Langmuir fit parameters $\Gamma_{\max }^{l}$ and $K_{c}$ of adsorption isotherms.

\begin{tabular}{ccc}
\hline Sample & $\Gamma_{\max }^{l}\left(\mathrm{mg} / \mathrm{m}^{2}\right)$ & $K_{c}$ \\
\hline S0 & $2.75 \pm 0.18$ & $0.14 \pm 0.09$ \\
S037 & $2.41 \pm 0.04$ & $0.28 \pm 0.07$ \\
S060 & $1.62 \pm 0.04$ & $0.54 \pm 0.07$ \\
S085 & $1.42 \pm 0.01$ & $0.94 \pm 0.05$ \\
\hline
\end{tabular}

Figure $11 \mathbf{b}$ ) shows the evolution of the maximum adsorbed amount $\Gamma_{\max }^{l}$ as a function of the C8TEO grafting ratio. $\Gamma_{\max }^{l}$ decreases when the grafting ratio increases. Overall it decreases by a factor of two from the bare silica to the highest grafting ratio. This observed decrease cannot be understood by simply considering the polymer/substrate affinity (for a constant polymer/solvent affinity), expressed in terms of the polymer/substrate distance in the 3D Hansen space. Indeed this distance decreases as the grafting ratio increases, which at first sight would correspond to an increased adsorption. The opposite trend is observed.

However, this evolution can actually be explained in terms of the $\chi_{S}$ parameter introduced in Section 2.2 (equation 6). In this set of measurements the polymer-solvent distance in the Hansen space remains constant while the solvent/substrate distance, which corresponds to the second factor $\left(\overrightarrow{\delta_{A}}-\overrightarrow{\delta_{S}}\right)$ in eq.6, decreases as silica is increasingly grafted with C8TEO. This leads to an overall decrease of the adsorption energy parameter $\chi_{S}$, which may indeed induce a decrease of the polymer adsorption. The adsorption parameter $\left(\chi_{S}\right)$ defined in equation 6 was computed for all systems. Values are reported in Table 7.

The maximum adsorbed amounts $\Gamma_{\max }^{l}$ obtained in this set of measurements are reported in Figure 12 as a function of the adsorption parameter $\chi_{S}$ calculated from the relative positions of the representative points of PS, cyclohexane and the treated silica grades, as shown in Figure 6. Both sets of data in which either the surface treatement of the silica or the nature of the solvent vary are reported.

It is clear from Figure 12 that there is strong correlation between the adsorbed polymer amount and the $\chi_{S}$ parameter. On the side of positive values of $\chi_{S}$ all points seem to fall on a unique curve and show a strong increase of the adsorbed amount $\Gamma_{\max }^{l}$ as $\chi_{S}$ 
Table 7: The adsorption parameter $\chi_{S}$ calculated with Eq. 6 for all systems from the HSP and HDP values used and/or determined previously.

\begin{tabular}{cccc}
\hline Polymer & solvent & silica grade & $\chi_{S}$ \\
\hline PS170 & cyclohexane & S0 & 5.532 \\
PS170 & Diethyl-Malonate & S0 & -1.767 \\
PS170 & 1-Chlorobutane & S0 & 1.051 \\
PS170 & Anisole & S0 & -0.2131 \\
PS170 & 1,4-Dichlorobutane & S0 & 0.1441 \\
\hline BR200 & cyclohexane & S0 & 2.169 \\
BR200 & 1,4-Dichlorobutane & S0 & -0.966 \\
\hline PS170 & cyclohexane & S037 & 5.344 \\
PS170 & cyclohexane & S060 & 4.432 \\
PS170 & cyclohexane & S085 & 4.305 \\
\hline
\end{tabular}

increases. The points at $\chi_{S}$ values close to zero (corresponding e.g. to Diethyl-malonate) seem to deviate with respect to the general trend. This may possibly correspond to the low $\chi_{S}$ regime in Figure 1, in which an increase of the adsorption was inferred at $\chi_{S}$ values close to the adsorption threshold. Though not discussed in ref., ${ }^{27}$ this regime predicted for long chains may correspond to the presence of very long loops, as the fraction of segments directly adsorbed on the surface shall be very small in this low $\chi_{S}$ situation. Note also that, as mentionned above, a slightly different definition of $\chi_{S}$ was used by Silberberg, ${ }^{27}$ leading to different absolute values of $\chi_{S}$.

\section{Conclusion}

Polymer-silica interactions play a key role when processing polymer matrices with submicrometric silica particles or aggregates. This is true both when processing in internal mixers and by solvent casting. In this later case, it is clear that polymer adsorption should be promoted in order to stabilize the particle dispersion. Due to the high surface polarity of silica, mainly due to the large density of silanols at the silica surface, surface treatments are often required in order to compatibilize silica filler particles and improve the dispersion state. Adsorption experiments of polystyrene and butadiene in organic solutions were performed on 


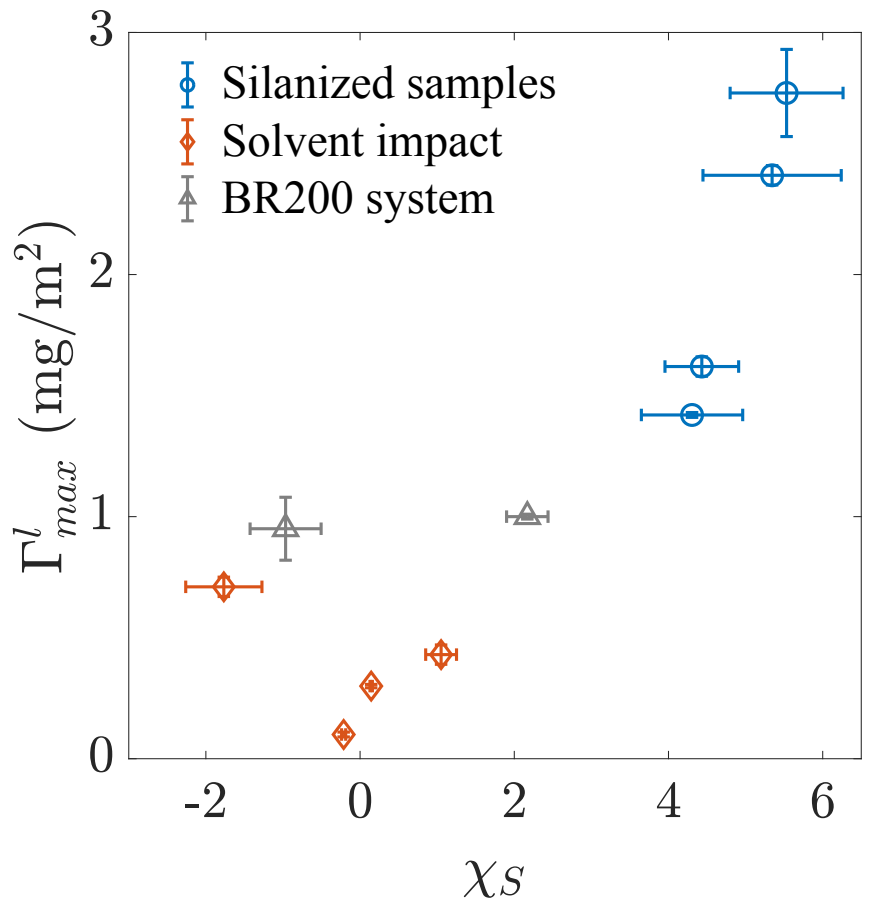

Figure 12: The maximum adsorbed amount $\Gamma_{\max }^{l}$ as a function of the adsorption parameter $\chi_{S}$.

Zeosil ${ }^{\circledR} 1165 \mathrm{MP}$ precipitated silica, either untreated or grafted with triethoxy(octyl)silane at different grafting ratios. It was shown that the choice of the solvent strongly impacts the polymer adsorption. As expected and already reported in the literature, one needs to use a near-theta solvent to promote polymer adsorption, while a good solvent promotes polymer solubiilization. Some residual adsorption, however, is observed in good solvent.

Specifically, a new approach based on the Hansen solubility concept is proposed. It is shown that dispersibility parameters can be determined based on measurements of the stability of silica suspensions in organic media, and silica may be effectively located in the 3D Hansen space.

Using the experimentally determined solubility and dispersibility parameters and proposed dispersibility regions relative position of polymer-solvent-silica can be now calculated. The unexpected decrease of polystyrene adsorption as silica is increasingly silanized is rationalized based on the consideration of an adsorption parameter $\chi_{S}$ computed from the relative 
locations of the solvent, polymer and particles in the 3D Hansen space. Basically, this parameter is related to the respective distances of the solvent and polymer representative points to that of the particle in Hansen space. This result and analysis actually illustrate the direct relationship between the Hansen parameters and the basic formalism of pair interactions as described e.g. in ref. ${ }^{41}$

The proposed approach developed in the context of silica nanoparticles may be extended to other nanocomposite materials and may concern other types of insoluble particles such as pigments in various application fields. It may enable predicting the impact of a surface treatment on the adsorption of a given polymer or on the stabilization of a dispersion. It

may as well enable the proper selection of an appropriate solvent in order to optimize a processing method.

\section{Supporting Information}

- The set of solvents used to determine HSP values of the polymers

- The set of solvents used to determine the HDP values of the silica grades

- Stability curves used to calculate the stability coefficients of silica suspensions

- 2D projections of convex hull corresponding to stability domains of the silica grades in the Hansen space

- Calculation of the molecular volumes

\section{References}

(1) Fu, S.; Sun, Z.; Huang, P.; Li, Y.; Hu, N. Some basic aspects of polymer nanocomposites: A critical review. Nano Materials Science 2019, 1, 2-30. 
(2) Voet, A.; Morawski, J. C.; Donnet, J. B. Reinforcement of Elastomers by Silica. Rubber Chemistry and Technology 1977, 50, 342-355.

(3) Alberola, N. D.; Benzarti, K.; Bas, C.; Bomal, Y. Interface effects in elastomers reinforced by modified precipitated silica. Polymer Composites 2001, 22, 312-325.

(4) Veiga, V. D.; Rossignol, T. M.; Crespo, J. D.; Carli, L. N. Tire tread compounds with reduced rolling resistance and improved wet grip. Journal of Applied Polymer Science $\mathbf{2 0 1 7}, 134,45334$.

(5) Bomal, Y.; Touzet, S.; Barruel, R.; Cochet, P.; Dejean, B. Developments in silica usage for decreased tyre rolling resistance. Kautschuk Gummi Kunststoffe 1997, 50, 434.

(6) Sarkawi, S. S.; Kaewsakul, W.; Sahakaro, K.; Dierkes, W. K.; Noordermeer, J. W. M. A Review on Reinforcement of Natural Rubber by Silica Fillers for Use in Low-Rolling Resistance Tyres. Journal of Rubber Research 2015, 18, 203-233.

(7) Ladouce-Stelandre, L.; Bomal, Y.; Flandin, L.; Labarre, D. Dynamic mechanical properties of precipitated silica filled rubber: Influence of morphology and coupling agent. Rubber Chemistry and Technology 2003, 76, 145-159.

(8) Guy, L.; Bomal, Y.; Ladouce-Stelandre, L. Elastomers reinforcement by precipitated silica. Kautschuk Gummi Kunststoffe 2005, 58, 43-49.

(9) Guy, L.; Daudey, S.; Cochet, P.; Bomal, Y. New Insights in the Dynamic Properties of Precipitated Silica Filled Rubber Using a New High Surface Silica. Kautschuk Gummi Kunststoffe 2009, 62, 383-391.

(10) Plueddemann, E. Silane Coupling Agents; Springer: New York, 1991.

(11) Parida, S. K.; Dash, S.; Patel, S.; Mishra, B. K. Adsorption of organic molecules on silica surface. Advances in Colloid and Interface Science 2006, 121, 77-110. 
(12) Pasternack, R. M.; Rivillon Amy, S.; Chabal, Y. J. Attachment of 3(Aminopropyl)triethoxysilane on Silicon Oxide Surfaces: Dependence on Solution Temperature. Langmuir 2008, 24, 12963-12971.

(13) Hasan, A.; Pandey, L. M. Kinetic studies of attachment and re-orientation of octyltriethoxysilane for formation of self-assembled monolayer on a silica substrate. Materials Science and Engineering: C 2016, 68, 423-429.

(14) Berriot, J.; Lequeux, F.; Monnerie, L.; Montes, H.; Long, D.; ; Sotta, P. Fillerelastomer interaction in model filled rubbers, a 1H NMR study. Journal of Non-Crystalline Solids 2002, 307-310, 719-724.

(15) Merabia, S.; Sotta, P.; ; Long, D. R. Heterogeneous nature of the dynamics and glass transition in thin polymer films. Eur. Phys. J. E 2004, 15, 189-210.

(16) Castellano, M.; Conzatti, L.; Costa, G.; Falqui, L.; Turturro, A.; Valenti, B.; Negroni, F. Surface modification of silica: 1 . Thermodynamic aspects and effect on elastomer reinforcement. Polymer 2005, 46, 695-703.

(17) Castellano, M.; Conzatti, L.; Turturro, A.; Costa, G.; Busca, G. Influence of the silane modifiers on the surface thermodynamic characteristics and dispersion of the silica into elastomer compounds. Journal of Physical Chemistry B 2007, 111, 4495-4502.

(18) Merabia, S.; Sotta, P.; Long, D. R. A Microscopic Model for the Reinforcement and the Nonlinear Behavior of Filled Elastomers and Thermoplastic Elastomers (Payne and Mullins Effects). Macromolecules 2008, 41, 8252-8266.

(19) Oberdisse, J. Structure and Rheological Properties of Latex-Silica Nanocomposite Films: Stress-Strain Isotherms. Macromolecules 2002, 35, 9441-9450.

(20) Tatou, M.; Genix, A.-C.; Imaz, A.; Forcada, J.; Banc, A.; Schweins, R.; Grillo, I.; 
Oberdisse, J. Reinforcement and Polymer Mobility in SilicaLatex Nanocomposites with Controlled Aggregation. Macromolecules 2011, 44, 9029-9039.

(21) Jouault, N.; Vallat, P.; Dalmas, F.; Said, S.; Jestin, J.; Bou, F. Well-Dispersed Fractal Aggregates as Filler in Polymer-Silica Nanocomposites: Long-Range Effects in Rheology. Macromolecules 2009, 42, 2031-2040.

(22) Chevigny, C.; Dalmas, F.; Di Cola, E.; Gigmes, D.; Bertin, D.; Bou, F.; Jestin, J. Polymer-Grafted-Nanoparticles Nanocomposites: Dispersion, Grafted Chain Conformation, and Rheological Behavior. Macromolecules 2011, 44, 122-133.

(23) Kawaguchi, M.; Hayakawa, K.; Takahashi, A. Adsorption of Polystyrene onto Silica at the Theta Temperature. Polymer Journal 1980, 12, 265-270.

(24) Kawaguchi, M.; Yamagiwa, S.; Takahashi, A.; Kato, T. Adsorption of polystyrene and poly(methyl methacrylate) onto a silica surface studied by the infrared technique. Comparison with theory. Journal of the Chemical Society, Faraday Transactions 1990, $86,1383-1387$.

(25) Silberberg, A. The adsorption of flexible macromolecules. Part I. The isolated macromolecule at a plane interface. The Journal of Physical Chemistry 1962, 66, 1872-1883.

(26) Silberberg, A. The adsorption of flexible macromolecules. Part II. The shape of the adsorbed molecule; the adsorption isotherm surface tension, and pressure. The Journal of Physical Chemistry 1962, 66, 1884-1907.

(27) Silberberg, A. Adsorption of Flexible Macromolecules. IV. Effect of SolventSolute Interactions, Solute Concentration, and Molecular Weight. The Journal of Chemical Physics 1968, 48, 2835-2851.

(28) de Gennes, P. G. Polymers at an interface; a simplified view. Advances in Colloid and Interface Science 1987, 27, 189-209. 
(29) Van der Beek, G. P.; Cohen Stuart, M. A.; Fleer, G. J.; Hofman, J. E. A chromatographic method for the determination of segmental adsorption energies of polymers. Polystyrene on silica. Langmuir 1989, 5, 1180-1186.

(30) Van der Beek, G. P.; Stuart, M. A. C.; Cosgrove, T. Polymer adsorption and desorption studies via proton NMR relaxation of the solvent. Langmuir 1991, 7, 327-334.

(31) Van der Beek, G. P.; Stuart, M. A. C.; Fleer, G. J.; Hofman, J. E. Segmental adsorption energies for polymers on silica and alumina. Macromolecules 1991, 24, 6600-6611.

(32) Fleer, G.; Cohen Stuart, M.; Scheutjens, J.; Cosgrove, T.; Vincent, B. Polymers at Interface; Chapman \& Hall, 1998.

(33) Dijt, J. C.; Cohen Stuart, M. A.; Fleer, G. J. Competitive Adsorption Kinetics of Polymers Differing in Length Only. Macromolecules 1994, 27, 3219-3228.

(34) Dijt, J. C.; Cohen Stuart, M. A.; Fleer, G. J. Kinetics of Adsorption and Desorption of Polystyrene on Silica from Decalin. Macromolecules 1994, 27, 3207-3218.

(35) Semenov, A. N.; Bonet-Avalos, J.; Johner, A.; Joanny, J. F. Adsorption of Polymer Solutions onto a Flat Surface. Macromolecules 1996, 29, 2179-2196.

(36) Radiom, M.; Maroni, P.; Borkovec, M. Influence of Solvent Quality on the Force Response of Individual Poly(styrene) Polymer Chains. ACS Macro Letters 2017, 6, 10521055.

(37) Schick, M. J.; Harvey, E. N. Interaction of Liquids at Solid Substrates; Advances in Chemistry; ACS, 1968; Vol. 87; Chapter 5, pp 63-71.

(38) Pekel, N.; Güven, O. Solvent, Temperature and Concentration Effects on the Adsorption of Poly(n-Butyl Methacrylate) on Alumina from Solutions. Turkish Journal of Chemistry 2002, 26, 221-228. 
(39) Linse, P. Effect of solvent quality on the polymer adsorption from bulk solution onto planar surfaces. Soft Matter 2012, 8, 5140-5150.

(40) Smith, P. E.; Mazo, R. M. On the Theory of Solute Solubility in Mixed Solvents. Journal of Physical Chemistry B 2008, 112, 7875-7884.

(41) Israelachvili, J. N. Intermolecular and Surface Forces - Third Edition; Elsevier, 2011.

(42) Ma, J.; Larsen, R. M. Comparative Study on Dispersion and Interfacial Properties of Single Walled Carbon Nanotube/Polymer Composites Using Hansen Solubility Parameters. ACS Applied Materials $\mathscr{G}$ Interfaces 2013, 5, 1287-1293.

(43) Launay, H.; Hansen, C. M.; Almdal, K. Hansen solubility parameters for a carbon fiber/epoxy composite. Carbon 2007, 45, 2859-2865.

(44) Arita, T.; Ueda, Y.; Minami, K.; Naka, T.; Adschiri, T. Dispersion of Fatty Acid Surface Modified Ceria Nanocrystals in Various Organic Solvents. Industrial \&6 Engineering Chemistry Research 2010, 49, 1947-1952.

(45) Wang, S.-H.; Liu, J.-H.; Pai, C.-T.; Chen, C.-W.; Chung, P.-T.; Chiang, A. S.T.; Chang, S.-J. Hansen solubility parameter analysis on the dispersion of zirconia nanocrystals. Journal of Colloid and Interface Science 2013, 407, 140-147.

(46) Hansen, C. M. Hansen Solubility Parameters: A User's Handbook; Second Edition; CRC Press, 1999.

(47) Ham, H. T.; Choi, Y. S.; Chung, I. J. An explanation of dispersion states of singlewalled carbon nanotubes in solvents and aqueous surfactant solutions using solubility parameters. Journal of Colloid and Interface Science 2005, 286, 216-223.

(48) SuB, S.; Sobisch, T.; Peukert, W.; Lerche, D.; Segets, D. Determination of Hansen parameters for particles: A standardized routine based on analytical centrifugation. Advanced Powder Technology 2018, 29, 1550-1561. 
(49) Lafaurie, A.; Azema, N.; Ferry, L.; Lopez-Cuesta, J.-M. Stability parameters for mineral suspensions: Improving the dispersion of fillers in thermoplastics. Powder Technology 2009, 192, 92-98.

(50) Raghavan, S. R.; Walls, H. J.; Khan, S. A. Rheology of Silica Dispersions in Organic Liquids: New Evidence for Solvation Forces Dictated by Hydrogen Bonding. Langmuir 2000, 16, 7920-7930.

(51) Flory, P. J. Thermodynamics of high polymer solutions. J. Chem. Phys 1942, 10, 51.

(52) Hildebrand, J.; Scott, R. Solubility of nanoelectrolites; Reinhold Publ. Corp.: New York, 1950.

(53) Hildebrand, J.; Scott, R. Regular solutions; Prentice-Hall, Inc, 1962.

(54) de Gennes, P. G. Scaling Concepts in Polymer Physics; Cornell University Press, 1979.

(55) Mansoori, G. A. A perturbation correction of the Flory-Huggins polymer solution theory. Condensed Matter Physics 2005, 42, 389-396.

(56) Hansen, C. M. The Three Dimensional Solubility Parameter - Key to Paint Component Affinities I. - Solvents, Plasticizers, Polymers, and Resins,. J. Paint Technol. 1967, 39, $104-117$.

(57) Hansen, C. M. The Three Dimensional Solubility Parameter - Key to Paint Component Affinities II. - Dyes, Emulsifiers, Mutual Solubility and Compatibility, and Pigments. J. Paint Technol. 1967, 39, 505-510.

(58) Hansen, C. M.; Skaarup; K. The three dimensional solubility parameter key to paint component affinities III. - Independent Calculation of the Parameter Components. J. Paint Technol. 1967, 39, 511-514. 
(59) Scheutjens, J. M. H. M.; Fleer, G. J. Statistical theory of the adsorption of interacting chain molecules. 1. Partition function, segment density distribution, and adsorption isotherms. The Journal of Physical Chemistry 1979, 83, 1619-1635.

(60) Scheutjens, J. M. H. M.; Fleer, G. J. Statistical theory of the adsorption of interacting chain molecules. 2. Train, loop, and tail size distribution. The Journal of Physical Chemistry 1980, 84, 178-190.

(61) Netz, R. R.; Andelman, D. Neutral and charged polymers at interfaces. Physics Reports 2003, 380, 1-95.

(62) Clark, A. T.; Robb, I. D.; Smith, R. Influence of solvent on the conformation of polymers adsorbed at the solid/liquid interface. Journal of the Chemical Society, Faraday Transactions 1: Physical Chemistry in Condensed Phases 1976, 72, 1489-1494.

(63) Buscall, R. The sedimentation of concentrated colloidal suspensions. Colloids and Surfaces 1990, 43, 33-53.

(64) Richardson, J.; Zaki, W. Sedimentation and fluidisation - 1. Institution of Chemical Engineers - Transactions 1954, 32, 35-52.

(65) Howell, J. S.; Stephens, B. O.; Boucher, D. S. Convex solubility parameters for polymers. Journal of Polymer Science Part B: Polymer Physics 2015, 53, 1089-1097. 


\section{For Table of Contents use only}

Competitive Adsorption between a Polymer and Solvents onto silica

J. Laurens, J. Jolly, G. Ovarlez, H. Fay, T. Chaussée, P. Sotta

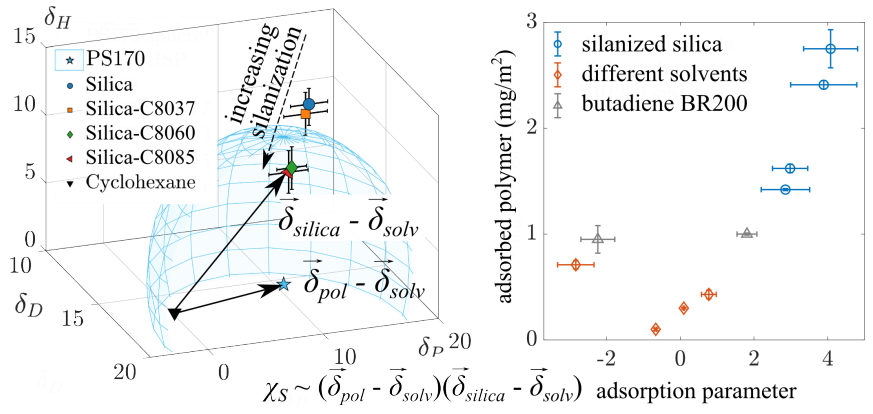

